ANL-6949

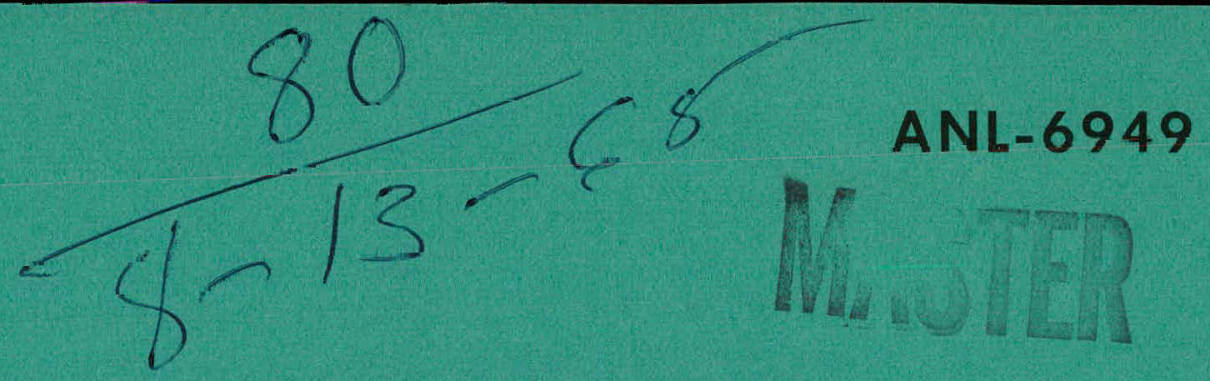

Argonne \ational Laboratoru

THEORETICAL ESTIMATES OF

PILE-UP DISTORTION

by

Raymond Gold 


\section{DISCLAIMER}

This report was prepared as an account of work sponsored by an agency of the United States Government. Neither the United States Government nor any agency Thereof, nor any of their employees, makes any warranty, express or implied, or assumes any legal liability or responsibility for the accuracy, completeness, or usefulness of any information, apparatus, product, or process disclosed, or represents that its use would not infringe privately owned rights. Reference herein to any specific commercial product, process, or service by trade name, trademark, manufacturer, or otherwise does not necessarily constitute or imply its endorsement, recommendation, or favoring by the United States Government or any agency thereof. The views and opinions of authors expressed herein do not necessarily state or reflect those of the United States Government or any agency thereof. 


\section{DISCLAIMER}

Portions of this document may be illegible in electronic image products. Images are produced from the best available original document. 


\section{LEGAL NOTICE}

This report was prepared as an account of Government sponsored work. Neither the United States, nor the Commission, nor any person acting on behalf of the Commission:

A. Makes any warranty or representation, expressed or implied, with respect to the accuracy, completeness, or usefulness of the information contained in this report, or that the use of any information, apparatus, method, or process disclosed in this report may not infringe privately owned rights; or

B. Assumes any liabilities with respect to the use of, or for damages resulting from the use of any information, apparatus, method, or process disclosed in this report.

As used in the above, "person acting on behalf of the Commission" includes any employee or contractor of the Commission, or employee of such contractor, to the extent that such employee or contractor of the Commission, or employee of such contractor prepares, disseminates, or provides access to, any information pursuant to his employment or contract with the Commission, or his employment with such contractor.

Printed in USA. Price $\$ 2.00$. Available from the Clearinghouse for Federal Scientific and Technical Information, National Bureau of Standards,

U. S. Department of Commerce, Springfield, Virginia 


\section{1.. ANं L"6949 \\ Physics \\ (TID-4500, 43rd Ed.)}

AEC Research and

Development Report

\section{ARGONNE NATIONAL LABORATORY \\ 9700 South Cass Avenue \\ Argonne, Illinois 60440.}

\section{THEORETICAL ESTIMATES OF \\ PILE-UP DISTORTION}

by

Raymond Gold

Reactor Physics Division

March 1965

Operated by The University of Chicago under

Contract W-31-.109-eng-38

with the

U. S. Atomic Energy Commission 


\section{TABLE OF CONTENTS}

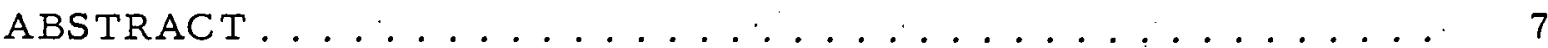

I. INTRODUCTION. ................ 7

II. GENERAL FORMULATION . . . . . . . . . . . . . . 9

A. Determination of the Baseline Probability Distribution ... 9

B. Formation of the Actual Pulse-height Distribution...... 13

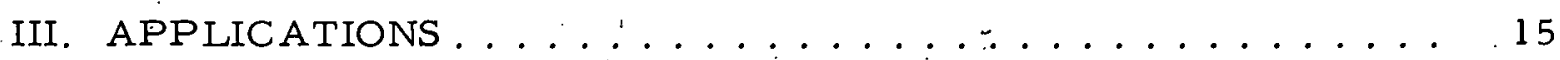

A. Case Ia. . . . . . . . . . . . . . . . 17

B. Case Ib. ...................... 18

C. Case Ic..................... 18

D. Case IIa ......................... 20

E. Case IIb ..................... 21

F. Case IIc .................... 21

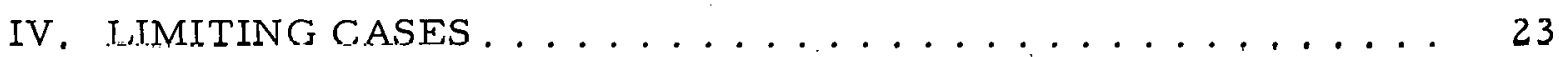

V. NUMERICAL RESULTS ................... 23

A. Case Ib....................... 24

B. Case IIa ...................... 26

C. Case Ic........................ 31

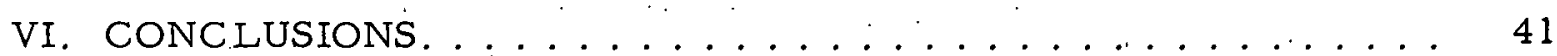

ACKNOW LEDGMENTS . . . . . . . . . . . . . . . . . 41

REFERENCES ......................... 42 


\section{LIST OF. FIGURES}

No.

Title

Page

1. Comparison of the Actual Pulse-height Distribution $D(\nu)$ for $\alpha=1,0.5$, and 0.1 in the Region $0 \leq \nu \leq 4$ with the Ideal Pulse-height Distribution $\mathrm{P}(\nu)=\mathrm{e}^{-\nu}$ (Case $\left.\mathrm{Ib}\right) \ldots \ldots \ldots$

2. Comparison of the Actual Pulse-height Distribution $D(\nu)$ for $\alpha=1,0.5,0.1$, and 0.005 in the Region $4 \leq \nu \leq 10$ with the Ideal Pulse-height Distribution $P(\nu)=e^{-\nu}$ (Case $\left.I b\right) \ldots \ldots 25$

3. Comparison of $\mathrm{E}(\nu)$ for Case Ib with the Simple Rossi-Staub Formula $(R-S)$ for $\alpha=1.0$ and $0.05 \ldots \ldots 26$

4. Comparison of $E(\nu)$ for Case Ib with the Simple Rossi-Staub Formula $(\mathrm{R}-\mathrm{S})$ for $\alpha=0.5$ and $0.01 \ldots \ldots \ldots$

5. The Actual Pulse-height Distribution, $D(\nu)$, for Case IIa with $\alpha=0.001,0.05$, and $1 \ldots \ldots \ldots \ldots \ldots$

6. The Actual Pulse-height Distribution, $D(\nu)$, for Case IIa with

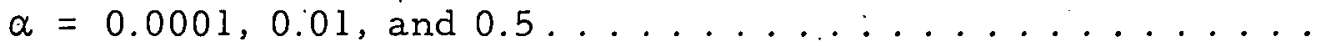

7. The Actual Pulse-height Distribution, $D(\nu)$, for Case IIa with



8. Comparison of $\mathrm{E}(\nu)=\mathrm{D}(\nu)$ for Case IIa with the Simple RossiStaub Formula. $(\mathrm{R}-\mathrm{S})$ for $\alpha=1.0$ and $0.001 \ldots \ldots \ldots$

9. Comparison of $E(\nu)=D(\nu)$ for Case IIa with the Simple RossiStaub Formula $(\mathrm{R}-\mathrm{S})$ for $\alpha=0.5$ and $0.005 \ldots \ldots \ldots$

10. Comparison of $D(\nu)$ for Case IIa with the Distribution of Souček [Ref. 7, Eq. (46)] for $\alpha=0.2$ and $1 \leq \nu \leq 1.9 \ldots \ldots$

11. Comparison of $D(\nu)$ for Case IIa with the Distribution of Souček [Ref. 7, Eq. (46)] for $\alpha=0.2$ and $1 \leq \nu \leq 7 \ldots \ldots$

12. Comparison of $D(\nu)$ for Case IIa with the Distribution of Souček [Ref. 7, Eq. (46)] for $\alpha=0.001$ and $1 \leq \nu \leq 4 \ldots \ldots$

13. The Actual Pulse-height Distribution, $D(\nu)$, for Case Ic with $\alpha=1.0$ and $\Delta=0.12$, Presented in Semilogarithmic Fashion. .

14. The Actual Pulse-height Distribution, $D(\nu)$, for Case Ic with $\alpha=1.0$ and $\Delta=0.12$, Presented in Linear Fashion . . . . . .

15. The Actual Pulse-height Distribution, $D(\nu)$, for Case Ic with $\alpha=1$ and $\Delta=0.06 \ldots \ldots \ldots \ldots \ldots \ldots \ldots \ldots \ldots \ldots \ldots \ldots . \ldots \ldots$

16. The Actual Pulse-height Distribution, $D(\nu)$, for Case Ic with $\alpha=1$ and $\Delta=0.01 .2 \ldots \ldots \ldots \ldots \ldots$ 


\section{LIST OF. FIGURES}

No.

Title

Page

17. Comparison of $[\triangle \cdot D(1)]$ as a Function of $\alpha$ with the Limiting Value $[\Delta \cdot P(1)]=(\pi)^{-1 / 2}$ for $\Delta=0.12,0.06$, and $0.012 \ldots \ldots$

18. Sum Peak Resolution. $r_{n}$ as a Function of $r$, the Resolution of the Ideal Pulse-height Distribution, for the Sum Peaks $n=2$,

- $3,4,5,6$, and $7 \ldots \ldots \ldots \ldots$

\section{LIST OF TABLES}

No.

Title

$\underline{\text { Page }}$

I. Special Case Designations ...................... 15

II. The Difference Function for Case Ib: $E(\nu)=D(\nu)-e^{-\nu} \ldots 25$

III. The Difference Function for Case IIa:

$$
E(\nu)=D(\nu) \quad(\nu>1) \ldots \ldots \ldots
$$

IV. The Difference Function for Case Ic:

$$
\begin{array}{ll}
E(\nu) \cong D(\nu) & (\nu>1.5) \\
\Delta=0.12 & (r=0.20)
\end{array}
$$

V. The Difference Function for Case Ic:

$$
\begin{array}{ll}
\mathrm{E}(\nu) \cong \mathrm{D}(\nu) & (\nu>1.5) \\
\Delta=0.06 & (\mathrm{r}=0.10)
\end{array}
$$

VI. The Difference Function for Case Ic:

$$
\begin{array}{ll}
E(\nu) \cong D(\nu) & (\nu>1.5) \\
\Delta=0.012 & (r=0.02) \ldots \ldots \ldots \ldots \ldots \ldots \ldots \ldots \ldots \ldots
\end{array}
$$




\section{THIS PAGE WAS INTENTIONALLY \\ LEFT BLANK}




\title{
THEORETICAL ESTIMATES OF PILE-UP DISTORTION
}

\author{
,by
}

Raymond Gold

\begin{abstract}
An analysis of pile-up distortion for a (pulse) detector is formulated by means of probability generating functions. Solutions are determined in the form of integral representations. Several important special cases, which are of interest in experimental applications, are treated. The representations for these special cases have been evaluated, and numerical results are presented in both graphical and tabular form. The results of the present method are compared with other estimates of pile-up distortion.
\end{abstract}

\section{INTRODUCTION}

The concept of resolving time for a detector is generally understood as some measure of the time duration of the detector response to a single event. Actually (in the present context), the resolving time can only be precisely defined in terms of the time interval between two successive events. It then may; be taken as the shortest interval of time for which the. second of the two successive events still gives rise to a detectable response. Although this second event may actually be detected, the information contained in this response can be seriously distorted. Thus, if a resolving time, $T$, is prescribed, then the detector response will be distorted if two or more events. occur within the order of the resolving time. These events will then be subject to misinterpretation. This latter effect; commonly called pile-up, has long been recognized as a distortion that can lead to erroneous experimental results.

To emphasize further the intimate relation between resolving time and pile-up distortion, one can examine the limit, $T \rightarrow 0$. In the examination of this limit, one must also require that the response amplitude diverge. Otherwise, the response would no longer correspond to a measurable result. Consequently; this limit implies a delta-function representation of the time-dependent response of the detector. Only in this ideal case can pile-up distortion be nonexistent. On the other hand, for all detection systems utilized in practice, this effect will be present and may, in certain instances, be an important source of experimental error. 
Considerable progress has been made in the experimental aspects of this problem. Electronic circuits have been developed to discriminate against pile-up events. $(1,2)$ These recent advances have been successful, and pile-up rejection techniques have come into widespread use. $(3,4)$

The theoretical treatment of this effect has not, however, received sufficient attention. Rossi and Staub(5) have considered the elementary case of a square-pulse response of constant amplitude. Pal $(6)$ has treated a more general case in the pile-up of alpha particles in a fission chamber. However, a more general formulation of this problem has not been available. Such an analysis is considered here. In the process of obtaining numerical results for important special cases of the present formulation, an alternate description of pile-up distorlion häs been given by Souček. (7-9) For certain special cases, the method described here and that of Soucek may complement each other. Souček obtains a solution in expansion form, whereas the present treatment results in an integral representation.

In terms of these treatments, a more quantitative judgment and comparison can be made of the discriminatory ability afforded by different rejection circuits. In addition, it is important to test the adequacy of the elementary case given by Rossi and Staub, (5) since it is customarily employed in present pile-up estimates. Of even more significance would be the availability of accurate estimates of the contribution that arises from this distortion. In many experiments, there may exist a high background of undesirable radiation, which is detected when the detection of relatively rare events, or other types of radiation, is actually desired. In such cir-. cumstances, reasonably accurate values of the pile-up count rate may be required. For many such measurements, a knowledge of pile-up distortion would prove helpful in both the preparation and analysis of experiments.

It will be convenient to introduce accepted experimental terminology in discussing this effect. Thus, the time dependence of the detector response will be referred to as the pulse shape of the detector. The distribution of the maxima in response amplitude (or the distribution of peak values) will be called the pulse-height distribution of the detector.

It is necessary to distinguish between two different types of pulseheight distributions that will be considered: those that contain pile-up distortion, and those that do not. These distributions will be designated as actual and ideal pulse-height distributions, respectively. An actual pulseheight distribution is one that arises from the output of a detector and therefore inherently contains pile-up distortion. In contrast, an ideal pulseheight distribution, which contains no pile-up distortion, is purely conceptual. That is', an ideal pulse-height distribution cannot occur in practice. It may be regarded as the pulse-height distribution that would arise if the detector possessed a delta-function pulse shape, or equivalently, as the pulse-height distribution that is assumed in the limit of vanishing count rate. 
In the present treatment, one regards both actual and ideal pulseheight distributions as probability distributions. The result of pile-up distortion is to (so-to-speak) transform an ideal probability distribution into an actual probability distribution. This transformation is formulated in essentially two basic steps. One first determines the probability distribution of the response amplitude level of the detector. It is appropriate to call this result the baseline probability distribution. The actual pulseheight distribution is then determined by a convolution of this baseline probability distribution and the ideal pulse-height distribution.

The general description of: obtaining pile-up estimates in this manner is given in the next section. The results of this description are then applied to several cases of interest. Computer programs have been developed to evaluate the integral representations that arise. Numerical results have been obtained, and comparisons are made with the simple Rossi-Staub model $(5)$ as well as with the results of Souček. (7-9)

\section{GENERAL FORMULLATION}

A. Determination of the Baseline Probability Distribution

The first basic step lies in the determination of the baseline probability distribution, $B(\nu)$. Here $B(\nu) d \nu$ represents the probability of finding the response amplitude level of the detector between $\nu$ and $\nu+\mathrm{d} \nu$. The method of probability generating functions will be employed to determine. $B(\nu)$. The theory and properties of probability'generating functions have been extensively treated by Feller. (10). Applications of probability generating functions to physical processes have been explored by Frisch. (11)

Let $Q(t, y)$ be the probability generating function that corresponds to the baseline probability distribution, $B(\nu)$. Here, $t$ represents the time variable, and $y$ is the dummy variable of the generating function. It.can be shown that $Q(t, y)$ satisfies the functional equation:(l l).

$$
Q(t+d t, y)=Q[t, A(y, t, d t)]: Q(d t, y)
$$

Equation (1) is based upon the fundamental property of probability generating functions. Namely, the probability generating function corresponding to the sum of two:random independent variables is given by the product of the probability generating functions corresponding to each.independent random variable.

In Eq. (1), the probability generating function $Q(d t, y)$ is related to the probability of the detection of another event in the interval dt. The function $A(y, t, d t)$ is also a probability generating function. It corresponds 
to the change in detector response during the time interval dt. Employing the chain rule for the partial derivative $\partial \mathrm{A} / \partial \mathrm{t}$, one can write

$$
\frac{\partial \mathrm{A}}{\partial \mathrm{t}}=\mathrm{g}\left(\mathrm{y}, \mathrm{t}, \tau_{\mathrm{i}}\right) \frac{\partial \mathrm{A}}{\partial \mathrm{y}}, \quad \mathrm{i}=1,2, \ldots, \mathrm{n}
$$

Here the change in $y$ during the interval dt is given by

$$
\text { dy }=\mathrm{g}\left(\mathrm{y}, \mathrm{t}, \tau_{\mathrm{i}}\right) \mathrm{dt}, \quad \mathrm{i}=1,2, \ldots, \mathrm{n} \text {. }
$$

It follows that the function $g\left(y, t, \tau_{i}\right)$ is related to the pulse shape of the detector, and the parameters $\tau_{i}, i=1,2, \ldots, n$, enter into the description of this pulse shape. The exact relation between $g\left(y, t, \tau_{i}\right)$ and the pulse shape of the detector, $F\left(t, \tau_{i}\right)$, will be developed later.

If $N(t)$ is the function describing the rate of detection of events as a function of time, then

$$
Q(d t, y)=1-N(t) d t+y N(t) d t
$$

Using Eqs. (2b) and (3) in Eq. (1) and retaining first-order terms only, one finds that $Q$ satisfies the equation

$$
\frac{\partial Q}{\partial t}=g\left(y, t, \tau_{i}\right) \frac{\partial Q}{\partial y}+(y-1) N(t) \dot{Q} .
$$

The more general case, wherein a nonconstant pulse-height distribution exists, may be handled by replacing the variable y introduced above, by the probability generating function corresponding to the ideal pulse-height dis tribution. Since ideal pulse-height distributions are continuous, it is natural to employ a continuous probability generating function. Thus, one would use

$$
y(x)=\int_{0}^{\infty} P(\nu) x^{\nu} d \nu,
$$

where $P(\nu)$ is the ideal pulse-height distribution. The normalization of $P(\nu)$ is chosen so that $y(x)$ satisfies

$$
y(1)=1
$$

Equation (6) is a boùndary condition required of all probability generating functions. In terms of the variables $(x, t)$, Eq. (4) becomes

$$
\frac{\partial Q}{\partial t}=g\left[y(x), t, T_{i}\right]\left(\frac{\partial y}{\partial x}\right)^{-1} \frac{\partial Q}{\partial x}+[y(x)-1] N(t) Q .
$$


Instead of treating Eq. (7) directly, it is simpler to deal with Eq. (4). One can then use the substitution defined by Eq. (5) in the solutions of Eq.. (4). This procedure corresponds to the rule for determining compound probability generating furictions. $(10,11)$

In most physical applications, the count-rate function $N(t)$ is not an explicit function of time, but $r$ ather a Poisson probability distribution. Thus, it is appropriate to replace $\mathrm{N}(\mathrm{t})$ by the mean value of this Poisson distribution. In addition, for many cases of interest, $g\left(y, t, \tau_{i}\right)$ may be as sumed independent of the time $t$. Such an assumption implies that the change of the detector response (or pulse shape) in an interval dt, can depend upon $\mathrm{dt}$, but not $\mathrm{t}$. This should be a reasonable approximation for many detection systems.

The above assumptions lead to a considerable simplification, since the formulation will then reduce to a time-independent description. Employing these assumptions, one can write

$$
N(t) \equiv N,
$$

and

$$
\mathrm{g}\left(\mathrm{y}, \mathrm{t}, \tau_{\dot{i}}\right) \equiv \mathrm{g}\left(\mathrm{y}, \tau_{\mathrm{i}}\right)
$$

As a result, Eq. (4) reduces to

$$
g\left(y, \tau_{i}\right) \frac{d Q}{d y}=(1-y) N Q
$$

The solution of Eq. (9) is

$$
Q(y)=Q_{0} \exp \left\{N \int^{y} \frac{(1-\omega)}{g\left(\omega, \tau_{i}\right)} d \omega\right\},
$$

where the constant $Q_{0}$ is determined by the usual boundary condition for. probability generating functions

$$
Q(1)=1
$$

The assumption introduced in Eq. (8b), that $g$ is independent of $t$, must depend intimately upon pulse shape. It is not difficult to examine the nature of the restriction introduced due to this assumption when $A(y, t)$ is a continuous probability generating function. In this event, $A(y, t)$ can be written in the form

$$
A(y, t)=y^{F\left(t, \tau_{i}\right)}
$$

where $F\left(t, \tau_{\mathrm{i}}\right)$ is the function describing the actual pulse shape of the detector. Hence, one has 


$$
\begin{aligned}
& \frac{\partial A}{\partial t}=y^{F(t, \tau)} F^{\prime}(t, \tau) \log y, \\
& \frac{\partial A}{\partial y}=y^{F(t, \tau)} \frac{F(t, \tau)}{y},
\end{aligned}
$$

and therefore

$$
g\left(y, t, \tau_{i}\right)=\frac{F^{\prime}(t, \tau)}{F(t, \tau)} \text { y } \log y .
$$

Using Eq. (8b) in Eq. (12c) implies

$$
\frac{F^{\prime}(t, \tau)}{F(t ; \tau)}=\beta
$$

where $\beta$ is a real constant. Consequently,

$$
F(t, \tau)=F_{0} \& \beta t
$$

with $F_{0}$ a real constant of integration. [The trivial solution $F(t, \tau) \equiv$ constant, which also fulfills.Eq. (8b), leads to $\mathrm{g}(\mathrm{y}, \dot{\tau}) \equiv 0$ : In this event, Eqs. $(10 a)$ and (10b) no longer provide an adequate description for the determination of $Q(y)$. However, the physical basis for the difficulty that arises with this trivial case is also apparent. Namely, the resulting pulse shape, $F(t, \tau) \equiv$ constant, can not correspond to any physical detection system. It follows, therefore, that this case is of no physical significance.] Thus, when $A(y, t)$ is a continuous probability generating function, only for $F(t, T)$ of the form given in Eq. (13b) will the time-independent formulation determine $Q(y)$. This limitation is not prohibitive, however, since many cases of practical importance can still be examined with the framework of the se assumptions.

In most cases of interest, $Q(x)$ will be a continuous probability generating function. Hence, the solution provided by Eq. (10) will determine an integral equation for the baseline probability distribution, $B(\nu)$. One has

$$
\mathrm{Q}[\mathrm{y}(\mathrm{x})]=\mathrm{Q}(\mathrm{x})=\int_{0}^{\infty} \mathrm{B}(\nu) \mathrm{x}^{\nu} \mathrm{d} \nu
$$

Equation (14) can often be solved by the method of Fourier transformis. With the transformation $x=e^{ \pm i \xi}, E q .(14)$. becomes

$$
Q_{ \pm}(\xi)=\int_{0}^{\infty} B(\nu) e^{ \pm i \nu \xi} d \nu
$$

Thus, the baseline probability distribution is given by 


$$
\mathrm{B}(\nu)=\frac{2}{\pi} \int_{0}^{\infty} \cos (\nu \xi) \mathrm{R}\left[Q_{ \pm}^{\prime}(\xi)\right] \mathrm{d} \xi
$$

or

$$
B(\nu)= \pm \frac{2}{\pi} \int_{0}^{\infty} \sin (\nu \xi) \operatorname{Irr}\left[Q_{ \pm}(\xi)\right] d \xi
$$

B. Formation of the Actual Pulse-height Distribution

The second basic step in the determination of $D(\nu)$, the actual pulseheight distribution, lies in properly combining the two probability distributions $B(\nu)$ and $P(\nu)$. To this end, it is convenient to introduce a new variable $\nu^{\prime}$ for the baseline probability distribution; $B\left(\nu^{\prime}\right)$. As before, $\nu^{\prime}$ represents the response-amplitude level of the detector, so that $B\left(\nu^{\prime}\right) d \nu^{\prime}$ is the probability of finding the response-amplitude level between $\nu^{\prime}$ and $\nu^{\prime}+\mathrm{d} \nu^{\prime}$. Since $P(\nu)$ is the ideal pulse-height distribution, $P(\nu)$ d $\nu$ is the (conceptual) probability of a response maximum or peak value between $\nu$ and $\nu+\mathrm{d} \nu$. It follows that the actual pulse-height or peak value is given by the sum $\nu+\nu^{\prime}$. Hence the actual pulse-height distribution is the probability distribution for the sum of the variables $\nu$ and $\nu^{\prime}$.

Thus, the actual pulse-height distribution, $D(\nu)$, can be obtained from a convolution of the baseline probability distribution and the ideal pulse-height distribution $\mathrm{P}(\nu)$. One can.write

$$
\mathrm{D}(\nu)=\int_{0}^{\infty} \mathrm{B}\left(\nu^{\prime}\right) \mathrm{P}\left(\nu-\nu^{\prime}\right) \mathrm{d} \nu^{\prime}
$$

Equation (17) is an application of the well-known theorem for forming the probability distribution of a sum of two independent variables: in terms of the probability distribution of each of the variables. (12)

Utilizing Eq. (16a) in Eq. (17), one? has

$$
\mathrm{D}(\nu)=\frac{2}{\pi} \int_{0}^{\infty} \int_{0}^{\infty} \cos \left(\nu^{\prime} \xi\right) \mathrm{R}\left[\mathrm{Q}_{ \pm}(\xi)\right] \mathrm{P}\left(\nu-\nu^{\prime}\right) \mathrm{d} \xi \mathrm{d} \nu^{\prime}
$$

If $\mathrm{P}(\nu)$ is unit-normalized, as implied in Eqs. (5) and (6), then one can show that $\mathrm{D}(\nu)$ is also unit-normalized. This result follows directly from condition $(10 \mathrm{~b})$. Consequently, one may interpret that both the actual and.ideal pulse-height distributions are probability distributions. That is, $P(\nu)$ represents the (conceptual) probability-for the occurrence of a maximum or 
peak value between $\nu$ and $\nu+d \nu$, whereas $D(\nu)$ is the actual probability of a detector response yielding a maximum or peak value between $\nu$ and $\nu+d \nu$, when one accounts for pile-up distortion.

Thus, the time-independent formulation leads to a solution that is an integral representation of $\mathrm{D}(\nu)$, viz., Eq. (18). It is therefore clear that even in the most idealized cases, the actual pulse-height distribution must intimately depend upon both the pulse shape and the ideal pulse-height distribution. Both of these quantities must be prescribed in order to define $D(\nu)$, the actual pulse-height distribution. This conclusion is clearly demonstrated by the special cases that are treated below.

A comparison of the present furnulation with the tricatmont of Souček $(7-9)$ is necessary. Souček determines the cumulants or semiinvariants of the baseline probabilty distribution, $B(\nu)$, utilizing an analysis originally given by Rice in the description of the Shot effect. (13) The semiinvariants of the actual pulse-height distribution, $D(\nu)$, are then found by summing the semi-invariants of $\mathrm{B}(\nu)$ and $\mathrm{P}(\nu)$. The actual pulse-height distribution $D(\nu)$ is then expanded in terms of the first four semi-invariants or (related) moments. Consequently, it is clear that this latter method and the present formulation employ different approximations. For example, while Souček's treatment yields only an expanded form: of the actual pulseheight distribution, it does not possess the limited applicability with regard to pulse shape that has been.discussed above for the present method. In view of the different approximations introduced, a comparison of the results obtained from these two methods is desirable. Such a comparison can be found in Cases Ic and IIa below. (See Section III for definitions of the special cases that have been treated.)

Finally, it must be pointed out that the actual pulse-height distribution, $D(\nu)$, can be expressed exactly in the integral representation form of Eq. (17). Instead of the approximate $B(\nu)$ obtained above, with the technique of probability generating functions, one can utilize the exact integral representation of $B(\nu)$ that has been given by Rice in the theory of the Shot effecl.* Moreover, using the latter result for $B(\nu)$ instead of Eqs. (16a) and (16b) provides the exact solution without the pulse-shape limitations of the present method.

In terms of more practical considerations, it may not be possible to directly use the exact solution in the determination of numerical results. Indeed, the exact integral representation of $B(\nu)$ is a considerably more complex Fourier transform that that given in Eqs. (16a) and (16b). Consequently, the possibility. that the exact solution will assume a tractable form that is suitable from a computational viewpoint is remote. The complexity of some of the approximate solutions given in the next section, and obtained within the framework of the present treatment, substantiate this contention.

*The exact form of $B(\nu)$ is given by Eq. (1.5-4) of Ref. 13. 


\section{APPLICATIONS}

In the applications that follow, only one dominant resolving time is introduced. Hence for these cases, one has $\tau_{i}=\tau, i=1,2, \ldots, n$. The special cases considered below are designated in Table I. Case I and Case II denote the two different pulse shapes that have been treated. In Case I (the square pulse shape), the time-dependent detector response can be written as

$$
\begin{array}{ll}
F(t, T)=1, & 0 \leq \mathrm{t} \leq \tau_{i} ; \\
F(\mathrm{t}, \tau)=0, & \mathrm{t}<0, \mathrm{t}>\tau .
\end{array}
$$

In Case II (the exponentially decaying pulse shape), one has

$$
\begin{array}{ll}
F(t, T)=e^{-t / T}, & t \geq 0 ; \\
F(t, T)=0, & t<0 .
\end{array}
$$

Table I

SPECIAL CASE DESIGNATIONS

\begin{tabular}{|c|c|c|}
\hline $\begin{array}{c}\text { Pulse } \\
\text { Pulse-height } \\
\text { Distribution }\end{array}$ & I. Square & II. Exponential \\
\hline (a) Delta Function & Ia & IIa \\
(b) Exponential & Ib & IIb \\
(c) Gaussian & Ic & IIc \\
\hline
\end{tabular}

For Case I, $\tau$ merely represents the width of the square pulse response of the detector. For Case II, $\tau$ represents the relaxation time for the decay of the response. In all of these special cases, one finds that the pile-up distortion is a function of the product of the average count rate, $N$, and the resolving time, $T$. Hence, it is convenient to define the single parameter

$$
\alpha=N \tau \text {. }
$$

The ideal pulse-height distributions utilized in Cases (a), (b), and (c) are given below in. Eqs. (22), (23), and (24), respectively. These distributions are unit-normalized in the interval $0 \leq \nu<\infty$. 


$$
\left.\begin{array}{ll}
\mathrm{P}(\nu)=\delta(\nu-1) ; & \\
\mathrm{P}\left(\nu-\nu^{\prime}\right)=\mathrm{e}^{-\left(\nu-\nu^{\prime}\right),} & \nu>\nu^{\prime} ; \\
\mathrm{P}\left(\nu-\nu^{\prime}\right)=0, & \cdot \nu^{\prime}>\nu ; \\
\mathrm{P}(0)=1 ; &
\end{array}\right\} .
$$

and

$$
P(\nu)=C \exp \left\{-\left(\frac{\nu-1}{\Delta}\right)^{2}\right\}, \quad \Delta>0
$$

and

$$
C=\left\{\frac{\sqrt{\pi}}{2} \Delta\left[1+\operatorname{Erf}\left(\Delta^{-1}\right)\right]\right\}^{-1},
$$

$$
\}
$$

where

$$
\operatorname{Erf}(s)=\frac{2}{\sqrt{\pi}} \int_{0}^{s} e^{-\omega^{2}} d \omega
$$

The distributions introduced above have not been chosen arbitrarily, but correspond to cases that often arise in practice. For Case (a), the delta function in Eq. (22) is the customary Dirac delta function and corresponds to an ideal pulse-height distribution with an integrable singularity at $\nu=1$. It follows that Case $\mathrm{Ia}$ is just the elementary case first considered by Rossi and Staub.(5) Equations (23a), (23b), and (23c) of Case (b) imply that we shall treat an exponentially-decreasing pulse-height spectrum. This. is typical of many background and noise spectra that arise in nuclear experiments. The condition that $\Delta>0$ in Eq. (24a) ensures that the Gaussian distribution employed in Case (c) is a representation of an experimental line spectrum. The relation between $\Delta$ and the experimental resolution $r$ (the relative full width at half maximum) is simply

$$
r=1.665 \Delta .
$$

Case (c) can arise, for example, when one deals with low-intensity lines in the presence of a single high-intensity transition.

The pulse-height scales for these ideal distributions have been chosen for computational convenience. Thus, in Case (c), the peak of the Gaussian distribution has been taken at $\nu=1$, and in Case (b), the exponential distribution possesses unit mean (i.e., $\bar{\nu}=1$ ). Hence, comparisons with experimental data will require, in addition to unit area normalization, the same pulse-height scale that has been chosen above. 
Each special case is analyzed separately below.

\section{A. Case Ia}

For the square pulse shape of Case I, defined in Eq. (19), the probability generating function $\mathrm{A}(\mathrm{y}, \mathrm{dt})$ is discrete. For Case Ia, the probability of a change in the detector response during the interval dt is $d t / T$. Hence, the probability generating function $A(y, d t)$ is given by

$$
A(y, d t)=y+[(1-y) / \tau] \cdot(d t) \text {. }
$$

A comparison of Eq. (26) with Eqs. (2a) and (12b) reveals that

$$
g(y, \tau)=(1-y) / \tau
$$

for Case I.

Using Eq. (27), the probability generating function determined from Eqs. (10a) and $(10 b)$ is

$$
Q(y)=e^{-\alpha} e^{\alpha y}
$$

For this simple case, one finds that the exact integral representation of Rice (13) can be reduced and, moreover, the resulting expression agrees precisely with that obtained in Eq. (28) above. Consequently, the results obtained for Case I will be exact.

The probability generating function $y(x)$ for Case Ia can be found by employing. Eq. (22) in Eq. (5). One finds

$$
y(\ddot{x}):=x
$$

Thus, one has

$$
Q(x)=e^{-\alpha} e^{\alpha x}
$$

as the probability generating function corresponding to the baseline probability distribution for Case Ia.

For this special case, it is apparent that the actual pulse-height distribution is discrete rather than continuous. One can, therefore, employ. the formal power-series expansion that is appropriate for a discrete probability generating function. With this representation

$$
Q(x)=e^{-\alpha} \sum_{n=0}^{\infty} \frac{(\alpha x)^{n}}{n !}
$$


one finds the probability $B_{n}$ is given by

$$
B_{n}=e^{-\alpha} \frac{\alpha{ }^{n}}{n !}, \quad n=0,1,2, \ldots
$$

Since the ideal pulse-height distribution is a delta function with a singularity at $\nu=1$, the actual pulse-height distribution is given by

$$
D_{n}=B_{n-1}=e^{-\alpha} \frac{\alpha(n-1)}{(n-1) !}, \quad n=1,2, \ldots \text {, }
$$

in agreement with the results obtained from elementary arguments. (5) Here the actual pulse-height distribution is also discrete and attains the values 1 unit, 2 units, ..., $n$ units, with the probabilities $D_{1}, D_{2}, \ldots, D_{n}$.

B. Case Ib

For Case $\mathrm{Ib}$, the probability generating function obtained from Eqs. (5) and (23) is

$$
y(x)=(1-\log x)^{-1}
$$

Using this result in Eq. (28), one finds

$$
Q(x)=\exp \left\{\frac{\alpha \log x}{1-\log x}\right\}
$$

The probability generating function given above can be employed in Eq. (18), together with the ideal distribution given in Eqs. (23a), (23b), and (23c). Thus, for Case $\mathrm{Ib}$; one finds the actual pulse-height distribution

$D(\nu)=\frac{2}{\pi} \int_{0}^{\infty}\left[\frac{\cos (\nu \xi)+\sin (\nu \xi)-e^{-\nu}}{1+\xi^{2}}\right] \cdot \exp \left(\frac{-\alpha \xi^{2}}{1+\xi^{2}}\right) \cdot \cos \left(\frac{\alpha \xi}{1+\xi^{2}}\right) \mathrm{d} \xi$.

C. Case Ic

The probability generating function obtained from Eqs. (5) and (24) is

$y(x)=\exp \left[\log x+(\Delta / 2)^{2}(\log x)^{2}\right] \cdot \frac{\left\{1+\operatorname{Erf}\left[\Delta^{-1}+(\Delta / 2) \log x\right]\right\}}{\left[1+\operatorname{Erf}\left(\Delta^{-1}\right)\right]}$.

Substituting this result into Eq. (28), one obtains 


$$
Q(x)=e^{-\alpha} \exp \left\{\alpha x\left[e[(\Delta / 2) \log x]^{2}\right] \frac{\left\{1+\operatorname{Erf}\left[\Delta^{-1}+(\Delta / 2) \log x\right]\right\}}{\left[1+\operatorname{Erf}\left(\Delta^{-1}\right)\right]}\right\} .
$$

Equations (24) and (38) can be utilized in Eq. (18), together with the transformation $x=e^{+i} \xi$. Employing analyticity arguments, the actual pulseheight distribution can be determined. One finds, for $[(\nu-1) / \Delta] \gg l$,

$$
\begin{aligned}
D(\nu)= & \frac{4 \mathrm{e}^{-\alpha}}{\pi\left[1+\operatorname{Erf}\left(\Delta^{-1}\right)\right]} \int_{0}^{\infty} \cos [(\nu-1) \xi] \mathrm{e}^{-(\Delta \xi / 2)^{2}} \\
& \exp \left\{\alpha \mathrm{e}^{-(\Delta \xi / 2)^{2}}\left[\cos \xi+\delta \int_{0}^{(\Delta \xi / 2)} \cdot \mathrm{e}^{\eta^{2}} \sin \left(\frac{2 \eta}{\Delta}-\xi\right) \mathrm{d} \eta\right]\right\} \\
& \cdot \cos \left\{\alpha \mathrm{e}^{-(\Delta \xi / 2)^{2}}\left[\sin \xi+\delta \int_{0}^{(\Delta \xi / 2)} \mathrm{e}^{\eta^{2}} \cos \left(\frac{2 \eta}{\Delta}-\xi\right) \mathrm{d} \eta\right]\right\} \mathrm{d} \xi
\end{aligned}
$$

with

$$
\delta=\frac{2}{\sqrt{\pi}} \frac{\exp \left(-\Delta^{-2}\right)}{\left[1+\operatorname{Erf}\left(\Delta^{-1}\right)\right]}
$$

Actually, we shall confine our attention to the domain $(0 \leq r \leq 0.2)$, which, according to Eq. (25), corresponds to the interval ( $0 \leq \Delta \leq 0.12$ ). For this range, one has $\delta \ll 1$ and $\operatorname{Erf}\left(\Delta^{-1}\right) \cong 1$. Neglecting all terms linear in $\delta$, Eq. (39a) reduces to*

$$
\begin{aligned}
D(\nu)= & \frac{2}{\pi} e^{-\alpha} \int_{0}^{\infty} e^{-(\Delta \xi / 2)^{2}} \cos [(\nu-1) \xi] \exp \left[\alpha e^{-(\Delta \xi / 2)^{2}} \cos \xi\right] . \\
& \cdot \cos \left[\alpha e^{-(\Delta \xi / 2)^{2}} \sin \xi\right] \mathrm{d} \xi .
\end{aligned}
$$

For the range of $\Delta$ considered above, Eq. (40). should provide adequate approximations of the actual pulse-height distribution for $\nu \gtrsim 1.5$.

The integral representation of $D(\nu)$, at $\nu=1$, assumes a form that is also tractable from a computational viewpoint. Namely, one has

*Since the domain under consideration includes (experimental) resolutions of up to $20 \%$, the subsequent results should apply to most detection systems used in spectral determinations. 
$\mathrm{D}(1)=\frac{\mathrm{e}^{-\alpha}}{\pi} \int_{0}^{\infty} \mathrm{e}^{-(\Delta \xi / 2)^{2}} \exp \left[\alpha \mathrm{e}^{-(\Delta \xi / 2)^{2}} \cos \xi\right] \cdot \cos \left[\alpha \mathrm{e}^{-(\Delta \xi / 2)^{2}} \sin \xi\right] \mathrm{d} \xi$.

This expression for $D(1)$ has been included since the value of $D(\nu)$ at $\nu=1$ in Case Ic is of definite experimental interest. The need for this point will be discussed more fully in Section $V$, which contains numerical results.

D. Case IIa

The exponentially decaying pulse shape, defined in Eq. (20), satisfies the condition given in Eq. (13b), which was derived for continuous probability generating functions. Consequently, Eq. (12c) can be àpplied, ănd fór Cáse II, on finds

$$
g\left(y, \tau_{i}\right)=-\frac{y \log y}{\tau}
$$

In this event, Eqs. (10a) and ( $10 b)$ yield

$$
Q(y)=e^{-\gamma \alpha} \frac{\exp \left[-\alpha E_{1}(-\log y)\right]}{(-\log y)^{\alpha}}
$$

where $E_{1}$ is the simple exponential integral,

$$
E_{1}(s)=\int_{s}^{\infty} \frac{e^{-\omega}}{\omega} d \omega
$$

and

$$
\gamma=0.5772157 \ldots
$$

is the Euler-Mascheroni constant. One can determine the actual pulseheight distribution by employing Eqs. (22) and (43) in Eq. (18). Using the transformation $x=e^{-i \xi}$, together with the resulting analyticity of the integrand, one finds

$D(\nu)=\frac{2}{\pi} e^{-\gamma \alpha} \int_{0}^{\infty} \frac{\cos [(\nu-1) \xi]}{\xi^{\alpha}} \exp [\alpha \operatorname{Ci}(\xi)] \cos [\alpha \operatorname{Si}(\xi)] d \xi$,

where $\operatorname{Si}(\xi)$ and $\mathrm{Ci}(\xi)$ are the customary sine and cosine integrals,

$$
\operatorname{Si}(\mathbf{s})=\int_{0}^{s} \frac{\sin \omega}{\omega} \mathrm{d} \omega
$$


and

$$
C i(s)=\int_{\infty}^{s} \frac{\cos \omega}{\omega} d \omega
$$

E. Case IIb

Here one need only employ the results of Case Ib and Case IIa. It follows from Eqs. (34) and (43) that $Q(x)$ for Case IIb is given by

$$
Q(x)=\frac{e^{-\gamma \alpha} \exp \left\{-\alpha E_{1}[\log (1-\log x)]\right\}}{[\log (1-\log x)]^{\alpha}}
$$

Equation (18) can again be evaluated by utilizing Eqs. (23a), (23b), (23c), and (47). The result for this case is

$$
\begin{aligned}
D(\nu)= & \frac{2}{\pi} e^{-\gamma \alpha} \int_{a}^{\infty}\left[\frac{\cos (\nu \xi)+\xi \sin (\nu \xi)-e^{-\nu}}{1+\xi^{2}}\right] \exp \left[-\alpha E_{1}(u)+\alpha e^{-u}\left\{\log \left[1+(v / u)^{2} \int \frac{\cos v}{2}+S(v / u, u)\right\}\right]\right. \\
& \cdot\left(u^{2}+v^{2}\right)^{-\alpha / 2} \cdot \cos \left[\alpha e^{-u}\left\{\log \left[1+(v / u)^{2}\right] \frac{\sin v}{2}-C(v / u, u)\right\}+\alpha \tan ^{-1}(v / u)\right] d \xi
\end{aligned}
$$

where

$$
\begin{aligned}
& u=\frac{1}{2} \log \left(1+\xi^{2}\right), \\
& v=\tan ^{-1}(\xi), \\
& S(v / u, u)=\int_{0}^{v / u} \sin (u \omega)\left[\frac{u}{2} \log \left(1+\omega^{2}\right)+\frac{1}{1+\omega^{2}}\right] d \omega, \\
& C(v / u, u)=\int_{0}^{v / u} \cos (u \omega)\left[\frac{u}{2} \log \left(1+\omega^{2}\right)+\frac{1}{1+\omega^{2}}\right] d \omega .
\end{aligned}
$$

F. Case IIc

Here the results of Case Ic and IIa may be employed. Using Eqs. (37) and (43), one has 
$Q(x)=\frac{e^{-\gamma \alpha} \exp \left[-\alpha E_{1}\left(-\log \mathbf{x}-[(\Delta / 2) \log \mathbf{x}]^{2}-\log \left\{\frac{1+\operatorname{Erf}\left[\Delta^{-1}+(\Delta / 2) \log \mathbf{x}\right]}{1+\operatorname{Erf}\left(\Delta^{-1}\right)}\right\}\right)\right]}{\left(-\log \mathbf{x}-[(\Delta / 2) \log \mathbf{x}]^{2}-\log \left\{\frac{1+\operatorname{Erf}\left[\Delta^{-1}+(\Delta / 2) \log \mathbf{x}\right]}{1+\operatorname{Erf}\left(\Delta^{-1}\right)}\right\}\right)^{\alpha}}$.

Utilizing the transformation $x=e^{-i \xi}$, with a treatment analogous to that of Case Ic, including the approximations therein, one finds, for $[(\nu-1) / \Delta]>>1$,

$$
\begin{aligned}
D(\nu) & =\frac{2}{\pi} e^{-\gamma \alpha} \int_{0}^{\infty} e^{-(\Delta \xi / 2)^{2}} \cos [(\nu-1) \xi]\left\{\exp \left[-\alpha E_{1}(u)+\alpha e^{-u}\left\{\log \left[1+(v / u)^{2}\right] \frac{\cos v}{2}+S(v / u, u)\right\}\right]\right\} \\
& \cdot\left(u^{2}+v^{2}\right)^{-\alpha / 2} \cdot \cos \left[\alpha e^{-u}\left\{\log \left[1+(v / u)^{2}\right] \frac{\sin v}{2}-c(v / u, u)\right\}+\alpha \tan ^{-1}(v / u)\right] d \xi,
\end{aligned}
$$

where

$$
u=(\Delta \xi / 2)^{2}
$$

and

$$
\mathrm{v}=\xi
$$

As in Case Ic; all terms linear in $\delta$ have been neglected. Within the domain we have considered for $\Delta, 0 \leq \Delta \leq 0.12$, Eq. (52) should also provide adequate approximations for $\nu \geqslant 1.5$. 


\section{LIMITING CASES}

Limiting forms of the cases treated above are of interest. These limiting cases provide necessary conditions that must be satisfied. The most significant of the se conditions, which arise from the physical requirements of our description, is given by

$$
\operatorname{Lim}_{\alpha \rightarrow 0} D(\nu)=P(\nu)
$$

Here the limit $\alpha \rightarrow 0$ can be implied by $N \rightarrow 0$ or $\tau \rightarrow 0$. The $\operatorname{limit} \tau \rightarrow 0$ has already been discussed in Section. I. The limit $N \rightarrow 0$, which also implies no pile-up distortion, is again a'physically-justifiable result. One can verify that condition (54) is satisfied by all the solutions presented above. This fact can also be established more generally, since one can prove that the integral representation of $\mathrm{D}(\nu)$, in the form of Eq. (18), directly satisfies this limiting condition.

Another limiting case of interest is that of $\Delta \rightarrow 0$ in Case (c). In this event, the ideal pulse-height distribution of Case (c) goes over to a delta function and thereby reduces to the ideal pulse-height distribution used in Case (a). Consequently, the limit $\Delta \rightarrow 0$ implies that the results of: Case (c) should reduce to the results.of Case (a). Indeed, one finds that Eq. (52) reduces to precisely Eq. (45) in the limit $\Delta \rightarrow 0$. For the comparison of Cases: Ia and Ic, it is more informative to compare the limiting form of the probability generating function, $Q(x)$, rather than the actual pulseheight distributions. This choice follows from the different interpretations utilized for the two actual pulse-height distributions, one as discrete and the other continuous, in these two cases, respectively. Employing $\Delta \rightarrow 0$ in Eq. (38) of Case Ic, one finds the probability generating function given in Eq. (30) for Case Ia. Consequently, this limiting condition is also satisfied.

\section{NUMERICAL RESULTS}

To obtain numerical results, it is necessary to evaluate the various integral representations given above. Computer programs have been developed that permit computation of $\mathrm{D}(\nu)$. The general method of evaluation utilized can most easily be explained in terms of the integral representation*

$$
D(\nu)=\int_{0}^{\xi_{M}} \mathrm{I}(\nu, \alpha, \Delta, \xi) \mathrm{d} \xi+\mathrm{J}\left(\xi_{M}\right)
$$

* The integral $\int_{0}^{\xi} \mathrm{M}(\nu, \alpha, \Delta, \xi) \mathrm{d} \xi$ in Eq. (55) has been evaluated by means either the trapezoidal rule or other convenient quadrature formulas. 
where $J\left(\xi_{M}\right)$ is an asymptotic expansion of the integral

$$
\int_{\xi}^{\infty} I_{a}(\nu, \alpha, \Delta, \xi) d \xi
$$

and $I_{a}(\nu, \alpha, \Delta, \xi)$ is the asymptotic form of the integrand $I(\nu, \alpha, \Delta, \xi)$.

The accuracy of this general method of evaluation was determined by employing different values of $\xi_{M}$. (All values of $\xi_{M}$ utilized were large enough to justify the asymptotic expansions introduced.) The values of $D(\nu)$ obtained in this manner were generally consistent to at least a few percent. However, the accuracy available does decrease when $D(\nu)$ becomes very small. Considerable variation exists for the lower bound of $D(\nu)$ corresponding to the above accuracy, for the different special cases treated below. As a consequence, the numerical results are restricted to a domain of values of $D(\nu)$ greater than a lower bound that generally lies between $10^{-5}$ and $10^{-8}$.

In certain special cases, it is advantageous to tabulate the difference between the actual and ideal pulse-height distributions. To this end, it is convenient to introduce the difference function

$$
E(\nu)=D(\nu)-P(\nu)
$$

The computer calculations obtained for Cases $\mathrm{Ib}$, ITa, and Ic are summarized below.

\section{A. Case $\mathrm{Tb}$}

For this case, $D(\nu)$ has been determined in the region: $0 \leq \nu \leq 10$. and $10^{-5} \leq \alpha \leq 1$. Figure 1 displays $D(\nu)(0 \leq \nu \leq 4)$ for $\alpha=1,0.5$, and 0.1 ; in comparison with $P(\nu)=e^{-\nu}$. Figure 2 presents similar data, but in the

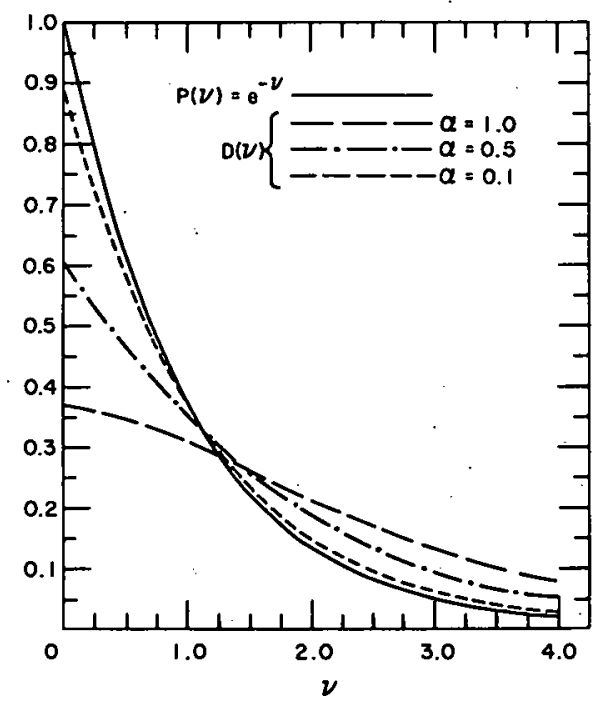

$112-4749$
Fig. 1

Comparison of the Actual Pulse-height Distribution $\mathrm{D}(\nu)$ for $\alpha=1,0.5$, and 0.1 in the Region $0 \leq \nu \leq 4$ with the Ideal Pulse-height Distribution $P(\nu)=\mathrm{e}^{-\nu}$ (Case Ib) 


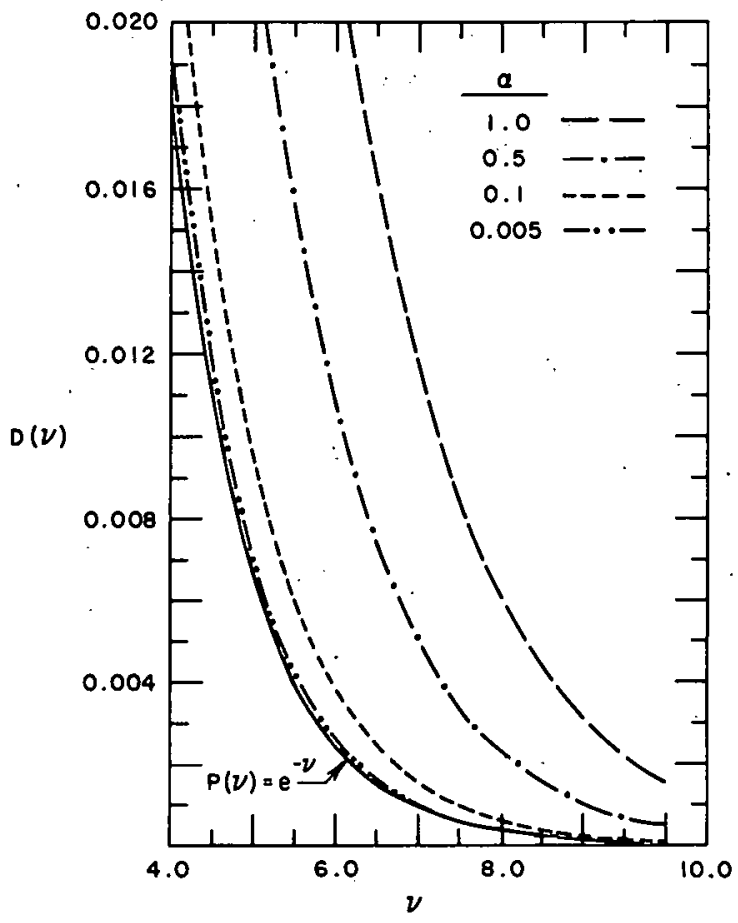

Fig. 2

Comparison of the Actual Pulseheight Distribution $D(\nu)$ for $\alpha=1,0.5,0.1$, and 0.005 in the Region $4 \leq \nu \leq 10$ with the Ideal Pulse-height Distribution $P(\nu)=e^{-\nu}$ (Case Ib)

interval $4 \leq \nu \leq 10$. The convergence $D(\nu) \rightarrow P(\nu)$ for $\alpha \rightarrow 0$, is evident. To emphasize this convergence behavior, the case $\alpha=0.005$ has also been included in Fig. 2. More extensive results are presented in Table II, where the difference function for Case Ib, $E(\nu)=D(\nu)-e^{-\nu}$, is tabulated.

Table II

THE DIFFERENCE FUNCTION FOR CASE ID

$E(v)=D(v)-e^{-v}$

\begin{tabular}{|c|c|c|c|c|c|c|c|c|c|}
\hline & 1 & 0.5 & 0.1 & 0.05 & 0.01 & 0.005 & 0.001 & 0.0001 & 0.00001 \\
\hline $\begin{array}{r}0.1 \\
0.2 \\
0.5 \\
1.0 \\
1.5 \\
2.0 \\
2.5 \\
3.0 \\
3.5 \\
4.0 \\
4.5 \\
5.0 \\
5.5 \\
6.0 \\
6.5 \\
7.0 \\
7.5 \\
8.0 \\
8.5 \\
9.0 \\
9.5 \\
10.0\end{array}$ & $\begin{array}{r}-5.38(-1) \\
-4.54(-1) \\
-2.57(-1) \\
-5.89(-2) \\
+3.71(-2) \\
7.67(-2) \\
8.64(-2) \\
8.15(-2) \\
7.05(-2) \\
5.79(-2) \\
4.60(-2) \\
3.56(-2) \\
2.70(-2) \\
2.04(-2) \\
1.50(-2) \\
1.10(-2) \\
7.96(-3) \\
5.74(-3) \\
4.11(-3) \\
2.93(-3) \\
2.08(-3) \\
1.47(-3)\end{array}$ & $\begin{array}{r}-3.28(-1) \\
-2.71(-1) \\
-1.40(-1) \\
-1.77(-2) \\
3.51(-2) \\
5.23(-2) \\
5.27(-2) \\
4.61(-2) \\
3.75(-2) \\
2.91(-2) \\
2.19(-2) \\
1.61(-2) \\
1.16(-2) \\
8.31(-3) \\
5.87(-3) \\
4.11(-3) \\
2.85(-3) \\
1.97(-3) \\
1.35(-3) \\
9.23(-4) \\
6.28(-4) \\
4.25(-4)\end{array}$ & $\begin{array}{r}-7.76(-2) \\
-6.25(-2) \\
-2.90(-2) \\
2.20(-4) \\
1.12(-2) \\
1.37(-2) \\
1.26(-2) \\
1.03(-2) \\
7.87(-3) \\
5.80(-3) \\
4.15(-3) \\
2.91(-3) \\
2.01(-3) \\
1.37(-3) \\
9.25(-4) \\
6.19(-4) \\
4.12(-4) \\
2.72(-4) \\
1.79(-4) \\
1.17(-4) \\
7.64(-5) \\
4.96(-5)\end{array}$ & $\begin{array}{r}-3.95(-2) \\
-3.16(-2) \\
-1.41(-2) \\
9.20(-4) \\
6.38(-3) \\
7.44(-3) \\
6.71(-3) \\
5.41(-3) \\
4.11(-3) \\
3.00(-3) \\
2.13(-3) \\
1.48(-3) \\
1.02(-3) \\
6.88(-4) \\
4.61(-4) \\
3.07(-4) \\
2.03(-4) \\
1.33(-4) \\
8.69(-5) \\
5.65(-5) \\
3.66(-5) \\
2.36(-5)\end{array}$ & $\begin{array}{r}-7.82(-3) \\
-5.99(-3) \\
-2.05(-3) \\
1.18(-3) \\
2.19(-3) \\
2.22(-3) \\
1.89(-3) \\
1.48(-3) \\
1.10(-3) \\
7.88(-4) \\
5.52(-4) \\
3.80(-4) \\
2.51(-4) \\
1.73(-4) \\
1.15(-4) \\
7.59(-5) \\
4.98(-5) \\
3.24(-5) \\
2.10(-5) \\
1.36(-5) \\
8.78(-6) \\
5.62(-6)\end{array}$ & $\begin{array}{r}-3.76(-3) \\
-2.73(-3) \\
-5.28(-4) \\
1.18(-3) \\
1.65(-3) \\
1.56(-3) \\
1.28(-3) \\
9.86(-4) \\
7.22(-4) \\
5.13(-4) \\
3.58(-4) \\
2.45(-4) \\
1.66(-4) \\
1.11(-4) \\
7.34(-5) \\
4.82(-5) \\
3.16(-5) \\
2.05(-5) \\
1.33(-5) \\
8.58(-6) \\
5.52(-6) \\
3.51(-6)\end{array}$ & $\begin{array}{r}-5.16(-4) \\
-1.23(-4) \\
6.67(-4) \\
1.21(-3) \\
1.21(-3) \\
1.02(-3) \\
7.96(-4) \\
5.87(-4) \\
4.20(-4) \\
2.95(-4) \\
2.02(-4) \\
1.37(-4) \\
9.20(-5) \\
6.10(-5) \\
4.01(-5) \\
2.63(-5) \\
1.72(-5) \\
1.11(-5) \\
7.16(-6) \\
4.60(-6) \\
2.96(-6) \\
1.88(-6)\end{array}$ & $\begin{array}{l}1.81(-4) \\
4.91(-4) \\
9.70(-4) \\
1.21(-3) \\
1.12(-3) \\
8.93(-4) \\
6.81(-4) \\
4.98(-4) \\
3.53(-4) \\
2.45(-4) \\
1.68(-4) \\
1.13(-4) \\
7.52(-5) \\
4.98(-5) \\
3.28(-5) \\
2.14(-5) \\
1.39(-5) \\
8.99(-6) \\
5.78(-6) \\
3.70(-6) \\
2.38(-6) \\
1.52(-6)\end{array}$ & $\begin{array}{l}2.71(-4) \\
4.91(-4) \\
9.70(-4) \\
1.21(-3) \\
1.09(-3) \\
8.80(-4) \\
6.73(-4) \\
4.88(-4) \\
3.47(-4) \\
2.40(-4) \\
1.63(-4) \\
1.11(-4) \\
7.36(-5) \\
4.88(-5) \\
3.20(-5) \\
2.09(-5) \\
1.36(-5) \\
.8 .79(-6) \\
5.66(-6) \\
3.63(-6) \\
2.32(-6) \\
1.47(-6)\end{array}$ \\
\hline
\end{tabular}

It is of interest to compare the present results with the simple Rossi-Staub formula of Case Ia. For these two cases, one has identical pulse shape but different pulse-height distributions. Figures 3 and 4 
compare $E(\nu)$ with interpolations based on Eq. (33) for $\alpha=\cdots 1.0$ and 0.05 and $\alpha=0.5$ and 0.01 , respectively. Pile-up estimates for these two cases can differ by many orders of magnitude. This comparison demonstrates that pile-up distortion is extremely sensitive to the ideal pulse-height distribution. It is apparent that the simple Rossi-Staub model possesses an extremely limited domain of validity.

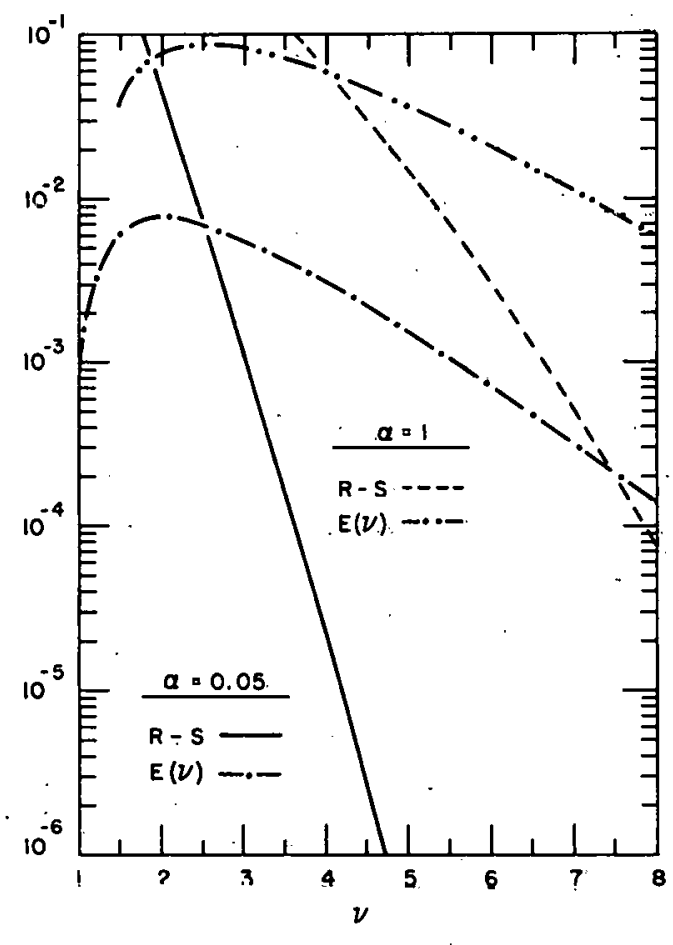

Fig. 3. Comparison of $E(\nu)$ for Case Ib with the Simple Rossi-Staub Formula (R-S) for $\alpha=1.0$ and 0.05



$112-4754$

Fig. 4. Comparison of $E(\nu)$ for Case Ib with the Simple Rossi-Staub Formula (R-S) for $a=0.5$ and 0.01

\section{B. Case IIa}

The integral representation of $\mathrm{D}(\nu)$ was evaluated for Case IIa in the range $10^{-5} \leq \alpha \leq 1$ for $\nu$-intervals $(\nu>1)$ corresponding to $D(\nu)>10^{-7}$. The actual probability distribution $D(\nu)$, for different values of $\alpha$, is depicted in Figs. 5, 6, and 7. The convergence criterion of Eq. (5.4) is again satisfied by these numerical results.

Some interesting additional features are also revealed by these curves. Discontinuities arise in the derivative of $D(\nu)$ at integral values of $\nu$. Moreover, these discontinuities becomes even more pronounced for decreasing $\alpha$. Note that the decrease in $D(\nu)$, from one "plateau" to the next (i.e., as $\nu$ increases across an integral value), is generally of the order of $\alpha$. In this manner, the results of the present case give qualitative 


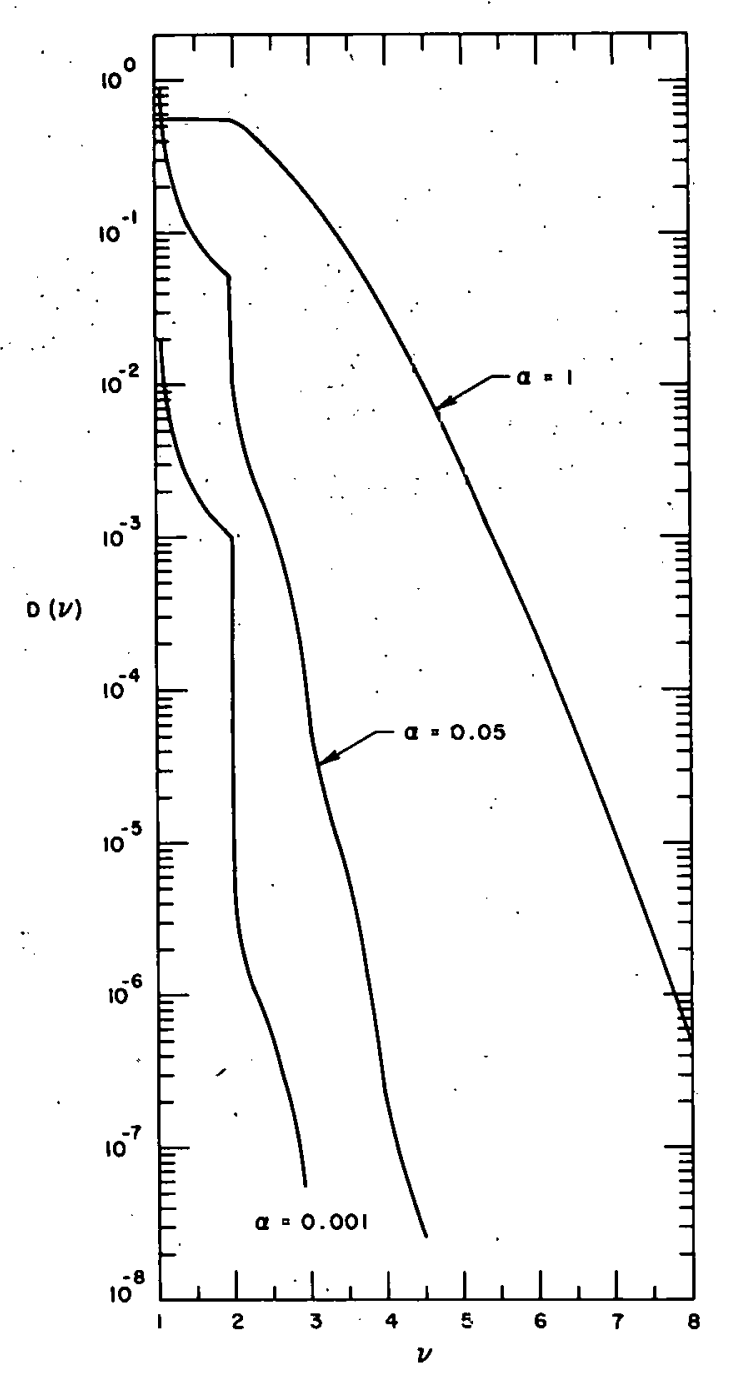

Fig. 5. The Actual Pulse-height Distribution, $D(\nu)$, for Case IIa with $\alpha=0.001$, 0.05 , and 1

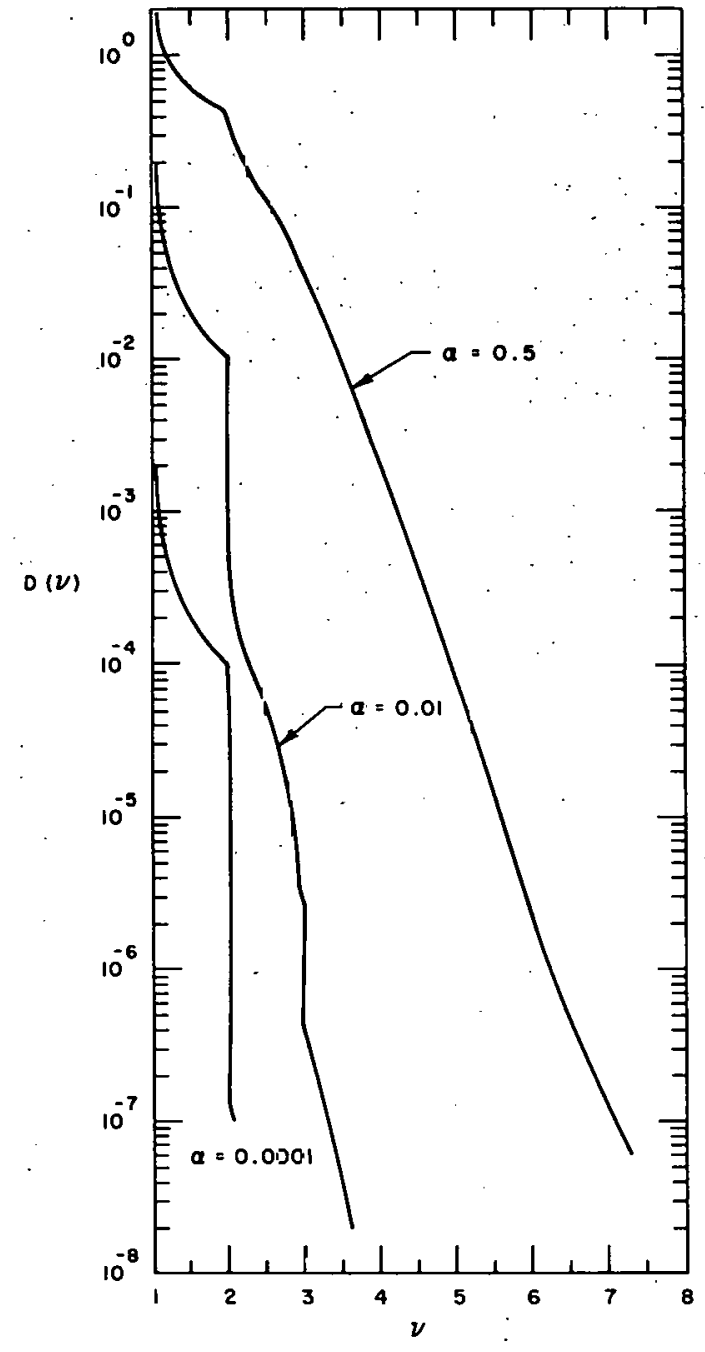

Fig. 6. The Actual Pulse-height Distribution, $D(\nu)$, for Case IIa with $\alpha=$

$0.0001,0.01$, and 0.5



Fig. 7. The Actual Pulse-height Distribution, $D(\nu)$, for Case IIa with $\alpha=$

$0.00001 ., 0.005$, and 0.10 
support to the concept of "n-fold" pile-up. Thus, the behavior displayed here should be compared with the simple Rossi-Staub result, Case Ia, Eq. (33), upon which the concept of. "n-fold" pile-up is based.

These similarities are; however, entirely qualitative since a direct comparison with Eq. (33) reveals pile-up estimates that can differ by orders of magnitude. To emphasize this conclusion, Figs. 8 and 9 compare $E(\nu)$ for Case IIa with interpolations based on the simple Rossi-Staub formula. These comparisons demonstrate that accurate estimates of pileup depend crucially upon pulse shape.* Since detectors usually possess an exponential decay type of response, rather than square pulse-shape response, the results of Case IIa may provide more accurate pile-up estimates for many experiments.

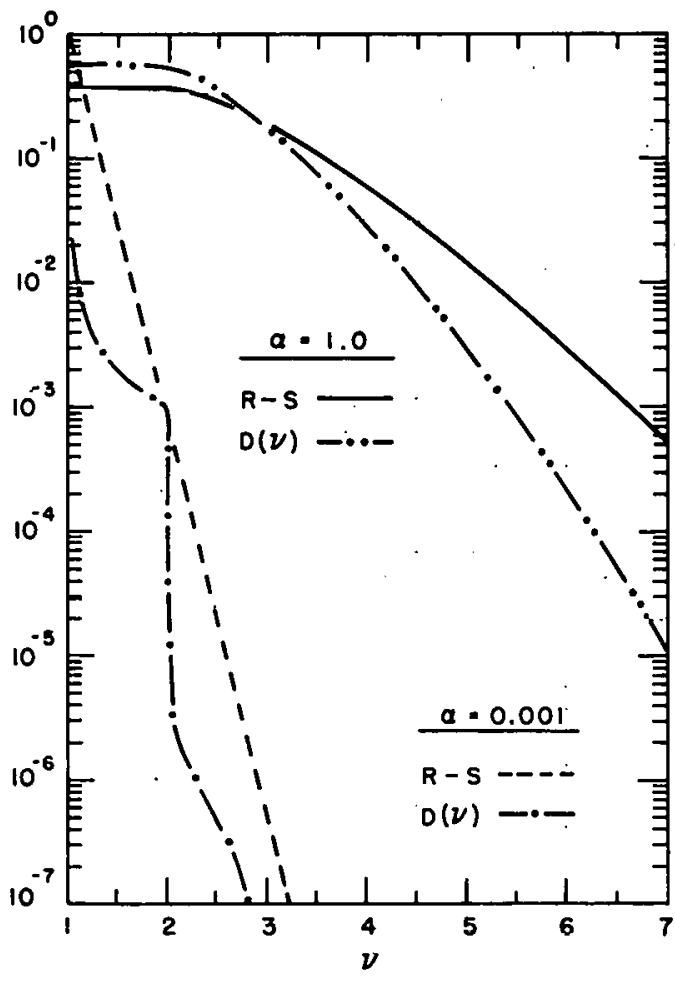

Fig. 8. Comparison of $\mathrm{E}(\nu)=$ $D(\nu)$ for Case IIa with the Simple Rossi-Staub Formula (R-S) for $\alpha=1.0$ and 0.001



$112-4753$

Fig. 9. Comparison of $E(\nu)=$ $D(\nu)$ for Case IIa with the Simple RossiStaub Formula ( $\mathrm{R}-\mathrm{S}$ ) for $\alpha=0.5$ and 0.005 .

Since $\mathrm{P}(\nu)=\delta(\nu-1)$ for Case IIa, then $\mathrm{E}(\nu)=\mathrm{D}(\nu)$ for $\nu>1$. Table III summarizes the results obtained for $\mathrm{D}(\nu)$ in Case IIa.

* The two cases compared here (viz., Cases Ia and IIa) have identical pulse-height distributions but differ in pulse shape. 
Table III

THE DIFFERETCE FUNCTION FOR CASE IIIa $E(v)-D(v) \quad(v>1)$

\begin{tabular}{|c|c|c|c|c|c|c|c|c|c|}
\hline 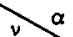 & 1.0 & 0.50 . & 0.10 & 0.05 & 0.01 & 0.005 & 0.001 & 0.0001 & 0.00001 \\
\hline 1.05 & - & $1.890 \quad(0)$ & $1.544 \quad(0)$ & $8.589(-1)$ & $1.942(-1)$ & $9.847(-2)$ & $1.993(-2)$ & $1.999(-3)$ & $1.999(-4)$ \\
\hline 1.1 & $5.615(-1)$ & $1.337(0)$ & $7.881(-1)$ & $4.448(-1)$ & $9.771(-2)$ & $4.943(-2)$ & $9.980(-3)$ & $9.998(-4)$ & $i .000(-4)$ \\
\hline 1.2 & - & $9.453(-1)$ & $4.175(-1)$ & $2.302(-1)$ & $4.920(-2)$ & $2.480(-2)$ & $4.992 .(-3)$ & $4.999(-4)$ & $5.000(-5)$ \\
\hline 1.3 & $5.615(-1)$ & $7.718(-1)$ & $2.932(-1)$ & $1.566(-1)$ & $3.293(-2)$ & $1.657(-2)$ & $3.329(-3)$ & $3.333(-4)$ & $3.333(-5)$ \\
\hline 1.4 & - & $6.684(-1)$ & $2.249(-1)$ & $1.192(-1)$ & $2.477(-2)$ & $1.244(-2)$ & $2.499(-3)$ & $2.500(-4)$ & $2.500(-5)$ \\
\hline 1.5 & $5.615(-1)$ & $5.979(-1)$ & $1.852(-1)$ & $9.640(-2)$ & $1.986(-2)$ & $9.965(-3)$ & $1.999(-3)$ & $2.000(-4)$ & $2.000(-5)$ \\
\hline 1.6 & - & $5.458(-1)$ & $1.563(-1)$ & $8.107(-2)$ & $1.658(-2)$ & $8.312(-3)$ & $1.666(-3)$ & $1.667(-4)$ & $1.667(-5)$ \\
\hline 1.7 & $5.614(-1)$ & $5.053(-1)$ & $1.368(-1)$ & $7.003(-2)$ & $1.423(-2)$ & $7.13(-3)$ & $.1 .428(-3)$ & $.1 .429(-4)$ & $1.429(-5)$ \\
\hline 1.8 & - & $4.727(-1)$ & $1.205(-1)$ & $6.168(-2)$ & $1.247(-2)$ & $6.243(-3)$ & $1.250(-3)$ & $1.250(-4)$ & $1.250(-5)$ \\
\hline 1.9 & $5.616(-1)$ & $4.456(-1)$ & $1.091(-1)$ & $5.515(-2)$ & $1.120(-2)$ & $5.553(-3)$ & $1.111(-3)$ & $1.121(-4)$ & $1.111(-5)$ \\
\hline 1.95 & - & $4.338(-1)$ & - & $5.238(-2)$ & $.1 .052(-2)$ & $5.261(-3)$ & $1.052(-3)$ & $1.052(-4)$ & $1.052(-5)$ \\
\hline 2.0 & - & $4.109(-1)$ & $7.124(-2)$ & $3.121(-2)$ & $5.264(-3)$ & $2.561(-3)$ & $5.007(-4)$ & $4.977(-5)$ & $4.946(-6)$ \\
\hline 2.05 & - & $3.211(-1)$ & $2.463(-2)$ & $6.639(-3)$ & $2.795(-4)$ & $7.170(-5)$ & $3.007(-6)$ & $9.814(-8)$ & $9.297(-10)$ \\
\hline 2.1 & $5.084(-1)$ & $2.776(-1)$ & $1.879(-2)$ & $4.966(-3)$ & $2.070(-4)$ & $5.299(-5)$ & $2.026(-6)$ & : & \\
\hline 2.2 & - & $2.186(-1)$ & $1.293(-2)$ & $3.262(-3)$ & $1.334(-4)$ & $3.341(-5)$ & $1.329(-6)$ & ? & : \\
\hline 2.3 & - & $1.767(-1)$ & $9.062(-3)$ & $2.296(-3)$ & $9.252(-5)$ & $2.314(-5)$ & $9.236(-7)$ & & \\
\hline 2.4 & - & $1.442(-1)$ & $6.736(-3)$ & $1.653(-3)$ & $6.561(-5)$ & $1.638(-5)$ & $6.461(-7)$ & · & : \\
\hline 2.5 & $3.339(-1)$ & $1.179(-1)$ & $4.871(-3)$ & $1.191(-3)$ & $4.652(-5)$ & $1.159(-5)$ & $4.597(-7)$ & & ${ }^{\circ}$ \\
\hline 2.6 & - & $9.602(-2)$ & $3.528(-3)$ & $8.439(-4)$ & $3.219(-5)$ & $8.026(-6)$ & $3.198(-7)$ & & \\
\hline 2.7 & - & $7.748(-2)$ & $2.476(-3)$ & $5.750(-4)$ & $2.141(-5)$ & $5.295(-6)$ & $2.115(-7)$ & & \\
\hline 2.8 & $2.315(-1)$ & $6.153(-2)$ & $1.635(-3)$ & $3.619(-4)$ & $1.282(-5)$ & $3.155(-6)$ & $1.219(-7)$ & & . \\
\hline 2.9 & - & $4.766(-2)$ & $9.704(-4)$ & $1.902(-4)$ & $5.973(-6)$ & $1.437(-6)$ & $5.535(-8)$ & & \\
\hline 2.95 & - & $4.137(-2)$ & $6.554(-4)$ & $1.166(-4)$ & $3.052(-6)$ & $7.118(-7)$ & & & \\
\hline 3.0 & - & $3.547(-2)$ & $4.063(-4)$ & $6.097(-5)$ & $2.561(-6)$ & - & & . & \\
\hline 3.05 & - & $3.066(-2)$ & - & $3.780(-5)$ & $3.093(-7)$ & $4.744(-8)$ & & & \\
\hline 3.1 & $1.463(-1)$ & $2.672(-2)$ & $2.433(-4)$ & $3.033(-5)$ & $2.406(-7)$ & $2.748(-8)$ & v & & \\
\hline 3.2 & - & $2.045(-2)$ & $1.629(-4)$ & $1.986(-5)$ & $1.598(-7)$ & $1.863(-8)$ & & & \\
\hline 3.3 & - & $1.567(-2)$ & $1.099(-4)$ & $1.308(-5)$ & $1.027(-7)$ & $1.322(-8)$ & & & \\
\hline 3.4 & - & $1.198(-2)$ & $7.318(-5)$ & $8.466(-6)$ & $6.564(-8)$ & & & & \\
\hline 3.5 & $7.3112(-2)$ & $9.095(-3)$ & $4.752(-5)$ & $5.315(-6)$ & $2.887(-8)$ & & & & \\
\hline 3.6 & - & - & c. $99 \varepsilon(-5)$ & $j \cdot i(1)(-6)$ & $1.735(-8)$ & · & & & \\
\hline 3.7 & - & - & $1.790(-5)$ & $1.740(-6)$ & & & & & \\
\hline 3.8 & $4.113(-2)$ &.- & $1.005(-5)$ & $8.708(-7)$ & & & & & \\
\hline 3.9 & - & - & $5.273(-6)$ & $3.807(-7)$ & & & & & \\
\hline 4.0 & $2.735(-2)$ & $2.004(-3)$ & $2.913(-6)$ & $1.778(-7)$ & & & & - & \\
\hline 4.1 & - & - & $1.866(-6)$ & $1.232(-7)$ & . & & & 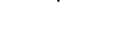 & \\
\hline 4.2 & - & - & $1.174(-6)$ & $7.208(-8)$ & & & & & \\
\hline 4.3 & - & - & $7.642(-7)$ & $5.677(-8)$ & & & & & \\
\hline 4.4 & - & - & $4.575(-7)$ & $3.579(-8)$ & & & & & \\
\hline 4.5 & $9.163(-3)$ & $4.132(-4)$ & $2.867(-7)$ & $2.554(-8)$ & & & & & \\
\hline 4.6 & - & - & $1.593(-7)$ & & & & & . & \\
\hline 4.7 & - & - & $9.717(-8)$ & & & & & & \\
\hline 4.8 & - & - & $4.793(-8)$ & & & & & & . \\
\hline 4.9 & - & - & $2.393(-8)$ & $\cdot$ & & & . & & \\
\hline 5.0 & $2.757(-3)$ & $7.595(-5)$ & & & & & & & \\
\hline 5.5 & $7.690(-4)$ & $1.308(-5)$ & & & & r & & & \\
\hline 6.0 & $1.987(-4)$ & $2 . \operatorname{nin}(-6)$ & & & & . & & & \\
\hline 6.5 & $4,823(-5)$ & $3.207(-7)$ & & & & & . & & \\
\hline 7.0 & $1.096(-5)$ & $9.152(-8)$ & & & & & & & \\
\hline 7.5 & $2.431(-6)$ & $.5 .788(-8)$ & & & & & . & . & \\
\hline 8.0 & $5.047(-7)$ & & & & & & & & \\
\hline 8.5 & $6.680(-8)$ & & r & & & & & & \\
\hline
\end{tabular}

The results for this case provide a basis for direct comparison with the work of Souček. (7) To this end, $D(\nu)$ has been determined in Case IIa for $\alpha=0.2$. Figures 10 and 11 compare the resulting $D(\nu)$ with the distribution given by Souček [viz., Eq. (46) of Ref. 7] for entirely equivalent as sumptions. Figure 10 is a detailed comparison for small $\nu$. The present 
results are roughly 25 to $50 \%$ lower than Souček's distribution for $\nu \widetilde{<} 1.4$. For $\ddot{1} .4 \leq \nu \leq 1.6$, the agreement is improved since these two distributions cross one another in the neighborhood of $\nu=1.5$. Beyond this cross-over point, $D(\nu)$ attains values that are roughly $50 \%$ higher than the results of Souček. In the region of larger $\nu$, depicted in Fig. 11 , there is another cross-over point in the neighborhood of $\nu=2.2$. Beyond this second crossover point, the disagreement becomes more significant.

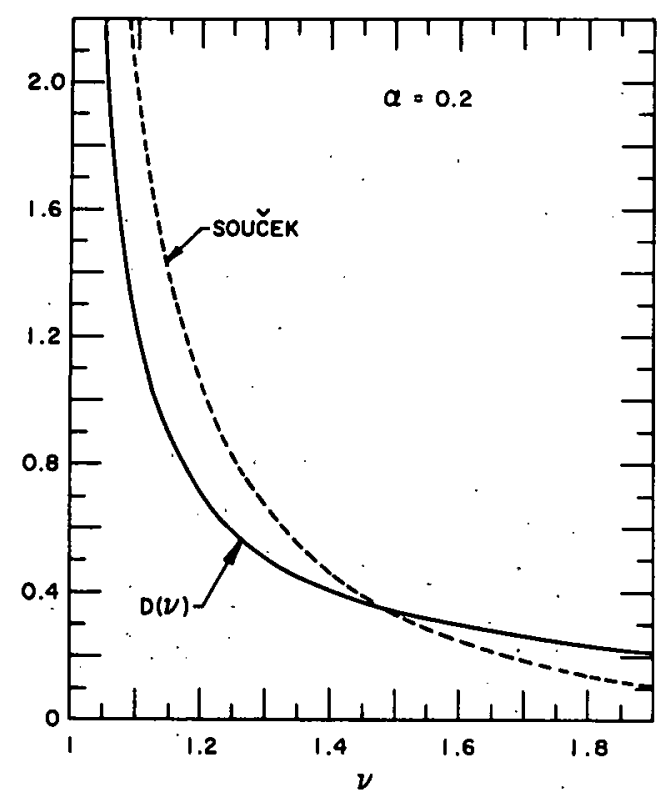

$112-4747$

Fig. 10. Comparison of $D(\nu)$ for Case IIa with the Distribution of Souček [Ref. 7. Eq. (46)] for $a=0.2$ and $1 \leq \nu \leq 1.9$

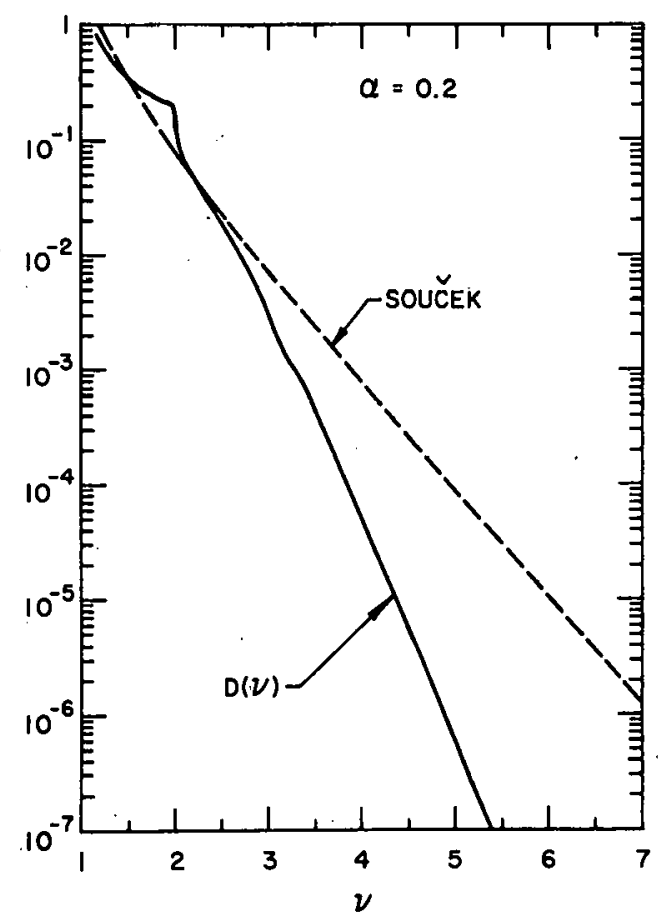

$112-4751$

Fig. 11. Comparison of $D(\nu)$ for Case IIa with the Distribution of Souček [Ref. 7, Eq. (46)] for $\alpha=0.2$ and $1 \leq \nu \leq 7$

This general behavior is not, however, unexpected. The expansion representation given by Souček must have a limited domain of validity and, in particular, must possess decreasing accuracy as $\nu$ increases. Furthermore, Souček's solution (corresponding to Case IIa) lacks the detail exhibited by the integral representation solution. For example, in the neighborhood of integral values of $\nu$, Souček's distribution possesses a continuous derivative in contrast with the discontinuities in the derivative of $D(\nu)$. While this behavior can be observed in Fig. 11, it becomes more striking for small $\alpha$. In this regard, Fig. 12 presents a comparison similar to that of Fig. 11, but for $\alpha=0.001$. As $\alpha$ decreases, marked and more serious differences develop between these two calculated distributions. 


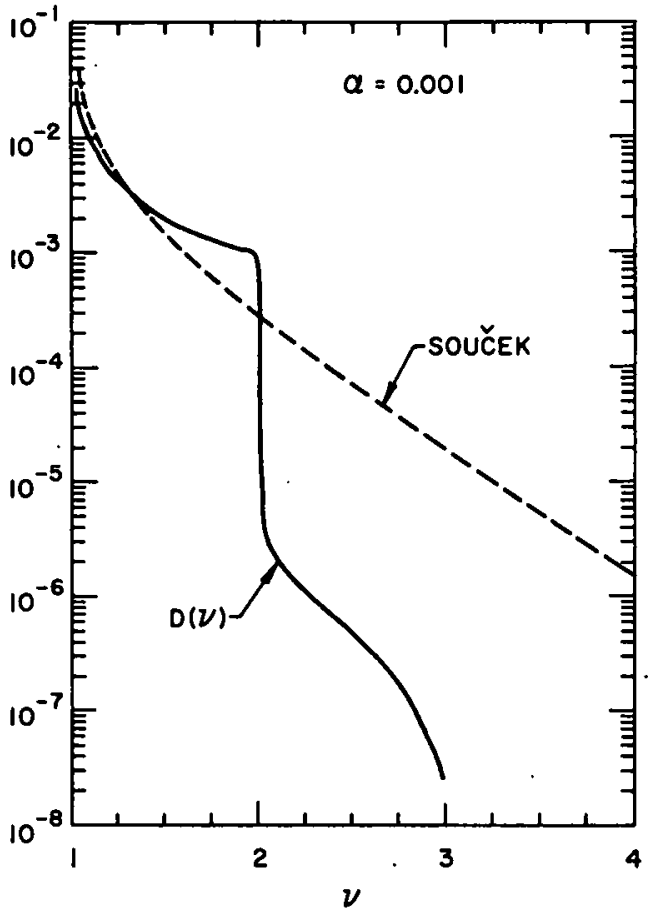

$112-4750$

Fig. 12. Comparison of $D(\nu)$ for Case IIa with the Distribution of Souček [Ref. 7, Eq. (46)] for $a=0.001$ and $1 \leq \nu \leq 4$
C. Case Ic

This case entails more extensive computational effort due to the existence of the additional parameter $\Delta$ as well as the detailed behavior of the resulting actual pulse-height distributions. The values of $\Delta$ that have been utilized are $\Delta=0.12$, 0.06 , and 0.012 , corresponding to resolutions of $\mathbf{r}=0.20,0.10$, and 0.02 , respectively. For each $\Delta$-value, $D(\nu)$ has been determined in the range $10^{-3} \leq \alpha \leq 1$ for a $\nu$-interval $(\nu>1.5)$ corresponding to $D(\nu) S 10^{-7}$.

The general behavior of the actual pulse-height distribution, $\mathrm{D}(\nu)$, is depicted in Figs. 13 and 14. These figures, which both correspond to $\alpha=1$ and $\Delta=0.12$, differ only in that Fig. 13 is a semilogarithmic display, whereas Fig. 14 presents the same data in a linear fashion. The "multiple Gaussians" or peaks that arise in $D(\nu)$ are striking. Each of the se "multiple Gaussians" is symmetrically centered about an integral value of $\nu$. It is convenient to refer to these "multiple Caussians" as n-fold sum peaks. Thus the ideal pulse-height distribution (i.e., the original Gaussian) is centered at $\nu=1$, the second sum peak at $\nu=2$, the third sum peak at $\nu=3$, and so on.

Fig. i 13

The Actual Pulse-height Distribution, $D(\nu)$, for Case Ic with $a=1.0$ and $\Delta=0.12$, Presented in Semilogarithmic Fashion

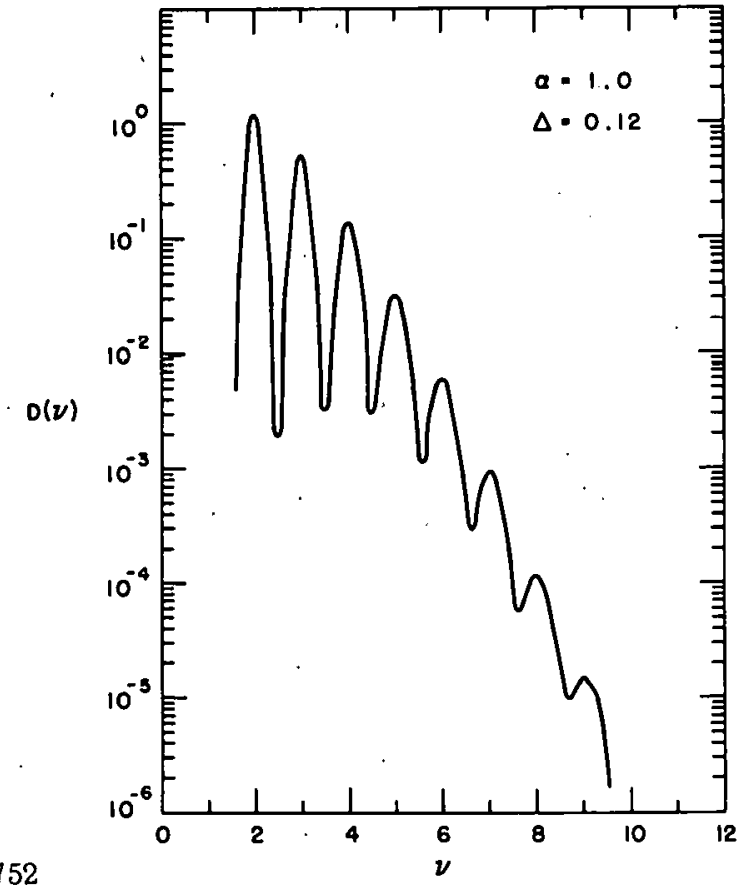






Fig. 14

The Actual Pulse-height Distribution, $\mathrm{D}(\nu)$, for Case Ic with $\alpha=1.0$ and $\Delta=0.12$, Presented in Linear Fashion

The behavior of the sum peaks for $\alpha=1$ and $\Delta=0.06$, and for $\alpha=1$ and $\Delta=0.012$ is displayed in Figs. 15 and 16, respectively. Comparison of Figs. 13, 15, and 16 reveals that the sum peaks become very narrow

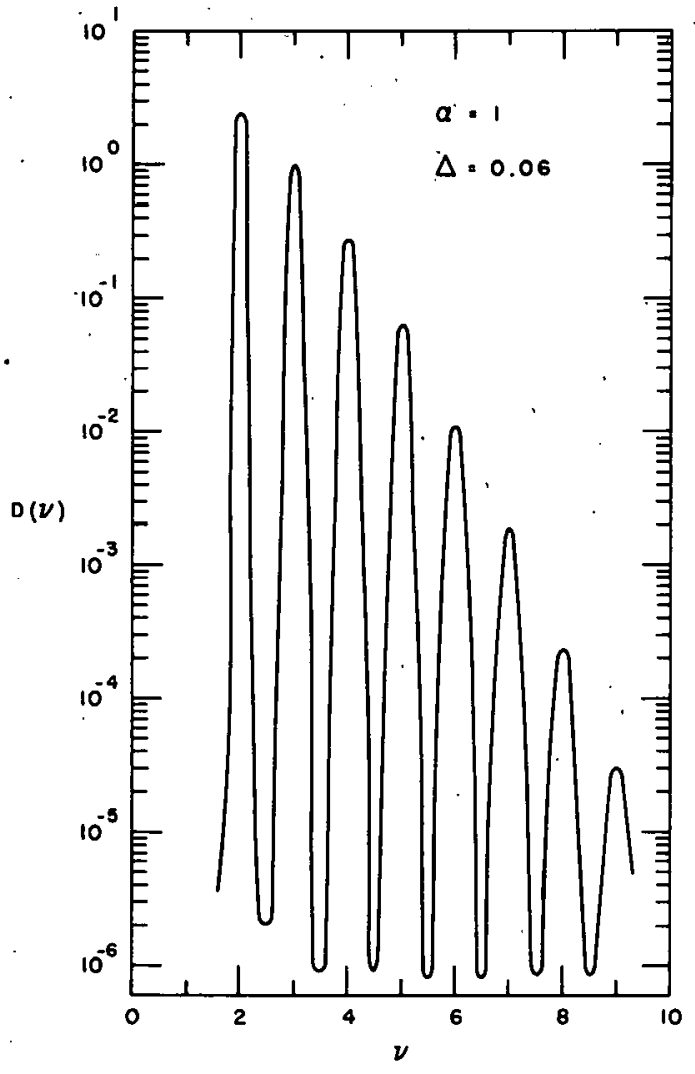

Fig. 15. The Actual Pulse-height Distri bution, $D(\nu)$, for Case Ic with $\alpha=1$ and $\triangle=0.06$

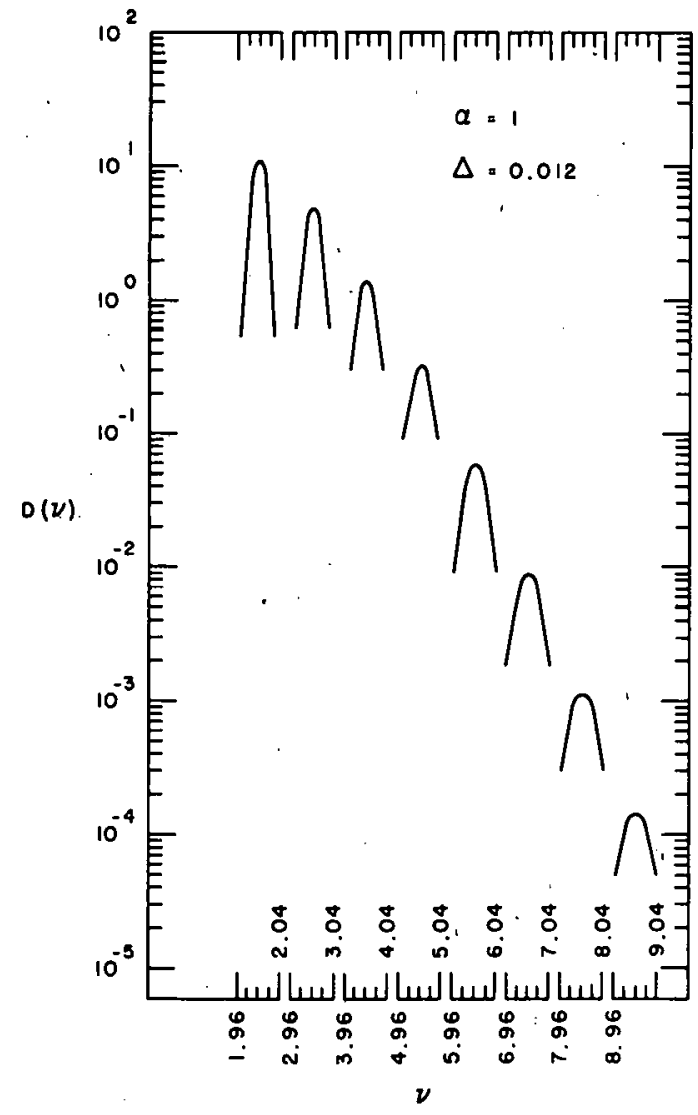

Fig. 16. The Actual Pulse-height Distribution, $D(\nu)$, for Case Ic with $a=1$ and $\triangle=0.012$ 
with decreasing $\Delta$, and this behavior confirms the convergence of Case Ic to the simple Rossi-Staub model for $\Delta \rightarrow 0$. Hence, for small $\Delta$, it is apparent that the sum-peak contributions are the only appreciable form of pile-up distortion. Consequently, the computations performed for $\Delta=0.012$ have been limited to defining the sum peaks in the neighborhood of integral. values of $\nu$. Tables IV, V, and VI contain a summary of the computational results for $D(\nu)$ in Case Ic. In view of earlier approximations, the range of accurate numerical results has been limited to $\nu>1.5$. Here $\mathrm{E}(\nu) ! \cong$ $\mathrm{D}(\nu)$, since one has $\mathrm{P}(\nu) \cong 0$ in this region.

Although the present treatment cannot define $D(\nu)$ in the neighborhood of $\nu=1$, the formulation of Souckek is applicable in this region. (9) Hence, for this special case, the two methods complement each other, and the present data can.be extended to include the neighborhood of $\nu=$ ' 1 . by employing. Souček's treatment.

An indirect measure of the distortion in the neighborhood of $\nu=1$ is available from the expression for $D(1)$ in Eq. (41). The difference between the actual and ideal pulse-height distributions can therefore be determined at $\nu=1$. Such a comparison is displayed in Fig. 17, where $[\Delta \cdots D(1)]$ is plotted as a function of $\alpha$ for $\Delta=0.12,0.06$, and 0.012 . Not only do these three curves coincide, but note the convergence $[\Delta \cdot D(1)] \rightarrow[\Delta \cdot P(1)]=$ $(\pi)^{-1 / 2}$, for $\alpha \rightarrow 0$. Since this result implies $[\Delta \cdot D(1)]$ is independent of $\Delta$ (at least for $\Delta \leq 0.12)$, Fig. 17 can be used to estimate $D(1)$ for all $\Delta \leq 0.12$. The value of $D(1)$ has been evaluated for every distribution computed in Case Ic. It can be obtained from the tabulated values of $E(1)$ given in Tables IV, V, and VI. The importance of this, point is that the value of $D(1)$ can serve as a convenient normalization value for experimental data [provided the pulse-height scale conforms with that chosen in Eq. (24a)].

Analysis of the data in Tables. IV,. V, and VI establishes three general rules for sum-peak behavior in Case Ic. The data obtained for $\mathrm{D}(\nu)$ demonstrate that in the region of experimental interest $(\alpha \leq 1$ and $\Delta \leq 0.12)$, each sum peak can be approximated by a Gaussian distribution: Consequently, in the neighborhood of integral values of $\nu ; D(\nu)$ can be written. in the form

$$
D_{n}(\nu)=p_{n} \exp \left\{-\left(\frac{\nu-n}{\Delta_{n}}\right)^{2}\right\} . n=2,3, \ldots
$$

Here $p_{n}$ is the peak height of the $n^{\text {th }}$ sum peak at $\nu=n$, and

$$
\mathrm{r}_{\dot{\mathrm{n}}}=1.665\left(\Delta_{\mathrm{n}} / \mathrm{n}\right), \quad \mathrm{n}=2,3, \ldots,
$$

is the resolution associated with the $n^{\text {th }}$ sum peak. Under these assumptions, one obtains the following rules:

(1) The resolution of a given sum peak, $r_{n}$, is independent of $\alpha$. 
Table IV

THE DIFFERENCE FUNCTION FOR CASE IC

$E(v) \cong D(v) \quad(v>1.5)$

$\Delta=0.12 \quad(r=0.20)$

\begin{tabular}{|c|c|c|c|c|c|c|c|}
\hline & 1.0 & 0.50 & 0.10 & 0.05 & 0.01 & 0.005 & 0.001 \\
\hline 1.0 & $-2,972$ & -1.850 & -0.448 & -0.229 & -0.047 & -0.024 & -0.005 \\
\hline 1.6 & $4.792(-3)$ & $3.898(-3)$ & $1.289(-3)$ & $6.113(-4)$ & $2.640(-4)$ & $6.398(-5)$ & $1.286(-5)$ \\
\hline 1.7 & $5.380(-2)$ & $4.430(-2)$ & $1.350(-2)$ & $6.947(-3)$ & $1.620(-3)$ & $7.258(-4)$ & $1.460(-4)$ \\
\hline 1.8 & $3.048(-1)$ & $2.514(-1)$ & $7.512(-2)$ & $3.943(-2)$ & $8.272(-3)$ & $4.124(-3)$ & $8.282(-4)$ \\
\hline 1.9 & $8.643(-1)$ & $7.125(-1)$ & $2.131(-1)$ & $1.117(-1)$ & $2.354(-2)$ & $1.169(-2)$ & $2.347(-3)$ \\
\hline 2.0 & 1.223 & 1.008 & $3.009(-1)$ & $1.581(-1)$ & $3.292(-2)$ & $1.654(-2)$ & $3.322(-3)$ \\
\hline 2.1 & $8.645(-1)$ & $7.125(-1)$ & $2.127(-1)$ & $1.117(-1)$ & $2.326(-2)$ & $1.169(-2)$ & $2.347(-3)$ \\
\hline 2.2 & $3.050(-1)$ & $2.514(-1)$ & $7.501(-2)$ & $3.943(-2)$ & $8.207(-3)$ & $4.125(-3)$ & $8.283(-4)$ \\
\hline 2.3 & $5.404(-2)$ & $4: 430(-2)$ & $1.321(-2)$ & $6.948(-3)$ & $1.447(-3)$ & $7.270(-4)$ & $1.472(-4)$ \\
\hline 2.4 & $4.658(-3)$ & $3.948(-3)$ & $1.129(-3)$ & $5.608(-4)$ & $1.273(-4)$ & $6.447(-5)$ & $1.287(-5)$ \\
\hline 2.5 & $1.927(-3)$ & $8.016(-4)$ & $9.508(-5)$ & $3.682(-5)$ & $6.484(-6)$ & $2.975(-6)$ & $1.052(-6)$ \\
\hline 2.6 & $1.230(-2)$ & $5.073(-3)$ & $3.100(-4)$ & $7.996(-5)$ & $3.098(-6)$ & $7.584(-7)$ & $6.914(-8)$ \\
\hline 2.7 & $6.188(-2)$ & $2.563(-2)$ & $1.574(-3)$ & $4.017(-4)$ & $1.708(-5)$ & $3.953(-6)^{\circ}$ & $1.890(-7)$ \\
\hline 2.8 & $1.976(-1)$ & $8.153(-2)$ & $4.846(-3)$ & $1.279(-3)$ & $5.274(-5)$ & $1.340(-5)$ & $7.334(-7)$ \\
\hline 2.9 & $3.959(-1)$ & $1.633(-1)$ & $9.784(-3)$ & $2.562(-3)$ & $1.074(-4)$ & $2.681(-5)$ & $1.193(-6)$ \\
\hline 3.0 & $4.995(-1)$ & $2.058(-1)$ & $1.232(-2)$ & $3.227(-3)$ & $1.349(-4)$ & $3.378(-5)$ & $1.369(-6)$ \\
\hline 3.1 & $3.964(-1)$ & $1.633(-1)$ & $9.805(-3)$ & $2.561(-3)$ & $1.072(-4)$ & $2.680(-5)$ & $1.088(-6)$ \\
\hline 3.2 & $1.980(-1)$ & $8.153(-2)$ & $4.905(-3)$ & $1.279(-3)$ & $5.279(-5)$ & $1.339(-5)$ & $5.695(-7)$ \\
\hline 3.3 & $6.229(-2)$ & $2.563(-2)$ & $1.500(-3)$ & $4.023(-4)$ & $1.712(-5)$ & $4.515(-6)$ & $1.787(-7)$ \\
\hline 3.4 & $1.279(-2)$ & $5.127(-3)$ & $3.054(-4)$ & $7.980(-5)$ & $3.685(-6)$ & $1.038(-6)$ & \\
\hline 3.5 & $3.355(-3)$ & $1.019(-3)$ & $5.635(-5)$ & i. $146(-5)$ & $2.549(-7)$ & & \\
\hline 3.6 & $9.272(-3)$ & $1.896(-3)$ & $3.254(-5)$ & $3.829(-6)$ & $7.177(-8)$ & & \\
\hline 3.7 & $3.056(-2)$ & $6.230(-3)$ & $6.606(-5)$ & $9.701(-6)$ & $9.721(-8)$ & & \\
\hline 3.8 & $7.174(-2)$ & $1.483(-2)$ & $1.6 \mathrm{I5}(-4)$ & $2.321(-5)$ & $2.058(-7)$ & & . \\
\hline 3.9 & $1.210(-1)$ & $2.497(-2)$ & $2.672(-4)$ & $3.923(-5)$ & $3.383(-7)$ & & \\
\hline 4.0 & $1.442(-1)$ & $2.970(-2)$ & $3.561(-4)$ & $4.665(-5)$ & $3.993(-7)$ & & \\
\hline 4.1 & $1.211(-1)$ & $2.497(-2)$ & $2.670(-4)$ & $3.943(-5)$ & $3.368(-7)$ & & \\
\hline 4.2 & $7.200(-2)$ & $1.483(-2)$ & $1.540(-4)$ & $2.334(-5)$ & $2.037(-7)$ & & \\
\hline 4.3 & $3.027(-2)$ & $6.232(-3)$ & $7.906(-5)$ & $1.083(-5)$ & $9.089(-8)$ & & \\
\hline 4.4 & $9.179(-3)$ & $1.870(-3)$ & $1.762(-5)$ & $3.732(-6)$ & & $\because$ & \\
\hline 4.5 & $2.898(-3)$ & $4.900(-4)$ & $4.469(-6)$ & $5.528(-7)$ & & & \\
\hline 4.6 & $3.826(-3)$ & $4.181(-4)$ & $2.381(-6)$ & $1.744(-7)$ & & & \\
\hline 4.7 & $9.322(-3)$ & $9.576(-4)$ & $2.536(-6)$ & $1.856(-7)$ & & & \\
\hline 4.8 & $1.851(-2)$ & $1.906(-3)$ & $4.535(-6)$ & $3.253(-7)$ & & & \\
\hline 4.9 & $2.805(-2)$ & $2.890(-3)$ & $6.919(-6)$ & $4.783(-7)$ & & & \\
\hline 5.0 & $3.219(-2)$ & $3.321(-3)$ & $7.945(-6)$ & $5.447(-7)$ & & & \\
\hline 5.1 & $2.805(-2)$ & $2.890(-3)$ & $7.063(-6)$ & $4.766(-7)$ & & & \\
\hline 5.2 & $1.850(-2)$ & $1.905(-3)$ & $4.056(-6)$ & $3.214(-7)$ & & & \\
\hline 5.3 & $9.446(-3)$ & $9.533(-4)$ & $2.445(-6)$ & $1.714(-7)$ & & & \\
\hline 5.4 & $3.606(-3)$ & $3.643(-4)$ & $5.870(-7)$ & . & . & & \\
\hline 5.5 & $1.343(-3)$ & $1.199(-4)$ & $1.463(-7)$ & & & & \\
\hline
\end{tabular}


Table IV (Contd.)

ITHE DIFFERENCE FUNCTIION FOR CASE IC

$$
\begin{aligned}
E(\nu) & \cong D(v) & & (v>1.5) \\
\Delta & =0.12 . & & (r=0.20)
\end{aligned}
$$

\begin{tabular}{|c|c|c|c|c|c|c|c|}
\hline$\nu \alpha$ & 1.0 & 0.50 & 0.10 & 0.05 & 0.01 & 0.005 & 0.001 \\
\hline $5.6^{\circ}$ & $1.211(-3)$ & $6.978(-5)$ & & & & & \\
\hline 5.7 & $2.171(-3)$ & $1.108(-4)$ & & & 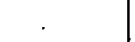 & & \\
\hline 5.8 & $3.736(-3)$ & $1.908(-4)$ & & & & . & \\
\hline 5.9 & $5.242(-3)$ & $2.706(-4)^{\prime}$ & & &. & . & \\
\hline 6.0 & $5.913(-3)$ & $3.033(-4)$ & $\theta$ & & . & & \\
\hline 6.1 & $5.242(-3)$ & $2.702(-4)$ & & . & - & & \\
\hline 6.2 & $3.760(-3)$ & $1.910 .(-4)$ & & & & & r \\
\hline 6.3 & $2.380(-3)$ & $1.072(-4)$ & & & $\because$ & & \\
\hline 6.4 & $1.011(-3)$ & $4.867(-5)$ & & & & & \\
\hline 6.5 & $4: 641(-4)$ & $1.885(-5)$ & & & & & \\
\hline 6.6 & $2.800(-4)$ & $9.142(-6)$ & & & & & . \\
\hline 6.7 & $3.922(-4)$ & $1.162(-5)$ & & & & & \\
\hline 6.8 & $6.133(-4)$ & $1.615(-5)$ & & & 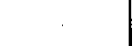 & & \\
\hline 6.9 & $8.237(-4)$ & $2.145(-5)$ & & & . & & $\cdot$ \\
\hline 7.0 & $9.138(-4)$ & $2.364(-5)$ & & & & $\cdot$ & \\
\hline 7.1 & $8.222(-4)$ & $2.144(-5)$ & & & . & & \\
\hline 7.2 & $6.250(-4)$ & $2.599(-5)$ & & & 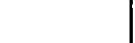 & & \\
\hline 7.3 & $3.743(-4)$ & $1.070(-5)$ & & & & & \\
\hline 7.4 & $1.920(-4)$ & & & & & & \\
\hline 7.5 & $9.083(-5)$ & & & & ' & & - \\
\hline 7.6 & $5.638(-5)$ & & & & & 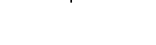 & \\
\hline 7.7 & $6.168(-5)$ & & & & & & \\
\hline 7.8 & $8.620(-5)$ & & & & & · & \\
\hline 7.9 & $1.127(-4)$ & & & & & . & \\
\hline 8.0 & $1.218(-4)$ & & & & & & - \\
\hline 8.1 & $1.115(-4)$ & & & & & & \\
\hline 8.2 & $8.454(-5)$ & & & & . & & $\therefore$ \\
\hline 8.3 & $5.643(-5)$ & & & & & & \\
\hline 8.4 & $3.244(-5)$ & & & & & $\cdot$ & \\
\hline 8.5 & $2.063(-5)$ & & & & & & • \\
\hline 8.6 & $1.107(-5)$ & & & & & & \\
\hline 8.7 & $9.683(-6)$ & & & & & & \\
\hline 8.8 & $1.190(-5)$ & & & & $\cdot$ & & \\
\hline 8.9 & $1.441(-5)$ & & & & & & r \\
\hline 9.0 & $1.556(-5)$ & & & & & & \\
\hline 9.1 & $1.473(-5)$ & & & & & & \\
\hline $9: 2$ & $1.235(-5)$ & & & & & & $\cdots$ \\
\hline 9.3 & $9.513(-6)$ & & & . & & & \\
\hline 9.4 & $5.085(-6)$ & & & 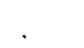 & & & \\
\hline 9.5 & $2.740(-6)$ & & & & & & \\
\hline
\end{tabular}


Table V

THE DIFFERENCE FUNCTION FOR CASE IC

$E(v) \cong D(v) \quad(v>1.5)$

$\Delta=0.06 \quad(r=0.10)$

\begin{tabular}{|c|c|c|c|c|c|c|c|}
\hline रे & 1.0 & 0.50 & 0.10 & 0.05 & 0.01 & 0.005 & .0 .001 \\
\hline 1.0 & -5.944 & -3.700 & -0.895 & -0.458 & -0.093 & -0.047 & -0.009 \\
\hline 1.6 & $3.705(-6)$ & $3.408,(-6)$ & $1.450(-6)$ & $6.254(-7)$ & $1.683(-7)$ & $1.070(-7)$ & $5.838(-8)$ \\
\hline 1.7 & $8.875(-6)$ & $7.093(-5)$ & $2.133(-5)$ & $1.146(-5)$ & $2.933\left(-6^{\prime}\right)$ & $1.813(-6)$ & $9.094(-7)$ \\
\hline 1.8 & $8.592(-3)$ & $7.049(-3)$ & $2.325(-3)$ & $1.230(-3)$ & $2.564(-4)$ & $1.290(-4)$ & $2.621(-5)$ \\
\hline 1.9 & $6.119(-1)$ & $5.028(-1)$ & $1.500(-1)$ & $7.884(-2)$ & 3. $.841(-2)$ & $0.247(-3)$ & $1.056(-3)$ \\
\hline 2.0 & 2.445 & 2.016 & $6.015(-1)$ & $3.162(-1)$ & $0.501(.2)$ & $3.207(-2)$ & โ. 64.1. $(-3)$ \\
\hline 2.1 & $6.121(-1)$ & $5.028(-1)$ & $1.500(-1)$ & $7.884(-2)$ & $1.641(-2)$ & $8.247(-3)$ & $1.656(-3)$ \\
\hline 2.2 & $8.898(-3)$ & $6.836(-3)$ & $2.326(-3)$ & $1.226(-3)$ & $2.552(-4)$ & $1.283(-4)$ & $2.586(-5)$ \\
\hline 2.3 & $9.242(-6)$ & $3.555(-5)$ & $7.840(-6)$ & $4.019(-6)$ & $8.951(-7)$ & $5.034(-7)$ & $3.478(-8)$ \\
\hline 2.4 & $2.273(-6)$ & $1.196(-6)$ & $2.903(-7)$ & $1.157(-7)$ & $2.647(-8)$ & $2.515(-8)$ & $8.290(-9)$ \\
\hline 2.5 & $2.068(-6)$ & $1.049(-6)$ & $2.479(-7)$ & $9.853(-8)$ & $2.249(-8)$ & $1.307(-8)$ & $6,986(-9)$ \\
\hline 2.6 & $3.482(-6)$ & $1.082(-6)$ & $2.224(-7)$ & $8.724(-8)$ & $1.937(-8)$ & $1.079(-8)$ & $5.940(-9)$ \\
\hline 2.7 & $2.397(-4)$ & $1.159(-4)$ & $6.091^{\circ}(-6)$ & $3.073(-6)$ & $4.059(-7)$ & $2.142(-7)$ & $2.151(-8)$ \\
\hline 2.8 & $2.460(-2)$ & $1.011(-2)$ & $6.075(-4)$ & $1.603(-4)$ & $6.908(-6)$ & $1.828(-6)$ & $1.397(-7)$ \\
\hline 2.9 & $3.959(-1)$ & $1.584(-1)$ & $9.729(-3)$ & $2.558(-3)$ & $1.067(-4)$ & $2.690(-5)$ & $1.147(-6)$ \\
\hline .3 .0 & $9.979(-1)$ & $4.092(-1)$ & $2.456(-2)$ & $6.454(-3)$ & $2.689(-4)$ & $6.763(-5)$ & $2.760(-6)$ \\
\hline 3.1 & $3.973(-1)$ & $1.639(-1)$ & $9.729(-3)$ & $2.557(-3)$ & $1.066(-4)$ & $2.687(-5)$ & $1.131(-6)$ \\
\hline 3.2 & $2.461(-2)$ & $9.697(-3)$ & $6.065(-4)$ & $1.598(-4)$ & $6.798(-6)$ & $1.765(-6)$ & $1.118(-7)$ \\
\hline 3.3 & $2.404(-4)$ & $1.083(-4)$ & $5.979(-6)$ & $2.319(-6)$ & $2.343(-7)$ & $1.142(-7)$ & $3.136(-9)$ \\
\hline 3.4 & $1.339(-6)$ & $5.699(-7)$ & $7.762(-8)$ & $3.601(-8)$ & $7.582(-9)$ & & \\
\hline 3.5 & $9.171(-7)$ & $3.868(-7)$ & $7.906(-8)$ & $3.074(-8)$ & $6.559(-9)$ & & \\
\hline 3.6 & $3.802(-6)$ & $1.246(-6)$ & $8.402(-8)$ & $2.993(-8)$ & $6.165(-9)$ & . & \\
\hline 3.7 & $5.563(-4)$ & $1.215(-4)$ & $2.473(-6)$ & $7.220(-7)$ & $7.517(-9)$ & & \\
\hline 3.8 & $1.796(-2)$ & $3.699(-3)$ & $4.509(-5)$ & $6.289(-6)$ & $5.518(-8)$ & & \\
\hline 3.9 & $1.446(-1)$ & $2.969(-2)$ & $3.549(-4)$ & $4.699(-5)$ & $3.936(-.7)$ & & \\
\hline 4.0 & $2.882(-1)$ & $5.939(-2)$ & $7.092(-4)$ & $9.356(-5)$ & $7.818(-7)$ & & \\
\hline 4.2 & $1.455(-1)$ & $2.966(-2)$ & $3.547(-3)$ & $4.692(-5)$ & $3.926(-7)$ & & \\
\hline 4.2 & $1.652(-2)$ & $3.697(-3)$ & $4.486(-5)$ & $6.176(-6)$ & $5.335(-7)$ & & . \\
\hline 4.3 & $5.570^{\circ}(-4)$ & $1.194(-4)$ & $1.405(-6)$ & $5.489(-7)$ & $6.459(-9)$ & & \\
\hline 4.4 & $5.873(-6)$ & $1.110(-6)$ & $4.539(-8)$ & $1.821(-8)$ & & & \\
\hline 4.5 & $9.426(-7)$ & $2.189(-7)$ & $3.297(-8)$ & $1.597(-8)$ & & & \\
\hline 4.6 & $8.475(-6)$ & $1.119(-6)$ & $3.379(-8)$ & $1.555(-8)$ & & & \\
\hline 4.7 & $4.330(-4)$ & $4.876(-5)$ & $1.448(-7)$ & $2.157(-8)$ & & & \\
\hline 4.8 & $5.680(-3)$ & $7.232(-4)$ & $2.314(-6)$ & $1.306(-7)$ & & & \\
\hline 4.9 & $3.697(-2)$ & $3.812(-3)$ & $9.697(-6)$ & $6.149(-7)$ & & & \\
\hline 5.0 & $6.445 \cdot(-2)$ & $6.641 \cdot(-3)$ & $1.591(-5)$ & $1.058(-6)$ & & & \\
\hline 5.1 & $3.778(-2)$ & $3.812(-3)$ & $9.635 \cdot(-6)$ & $6.137(-7)$ & & & \\
\hline 5.2 & $5.539(-3)$ & $7.227 \cdot(-4)$ & $2.239(-6)$ & $1.284(-7)$ & & & \\
\hline 5.3 & $4.348(-4)$ & $4.806(-5)$ & $6.226(-7)$ & $1.896(-8)$ & & & \\
\hline 5.4 & $8.454(-6)$ & $1.073(-6)$ & & & & & \\
\hline 5.5 & $8.219(-7)$ & & & & & & \\
\hline
\end{tabular}


Table V (Contd.)

THE DTFFERENCE FUNCTION FOR CASE I $c$

$$
\begin{array}{rlrl}
E(v) \simeq D(v) & & (v>1.5) \\
: \Delta & =0.06 & & (r=0.10)
\end{array}
$$

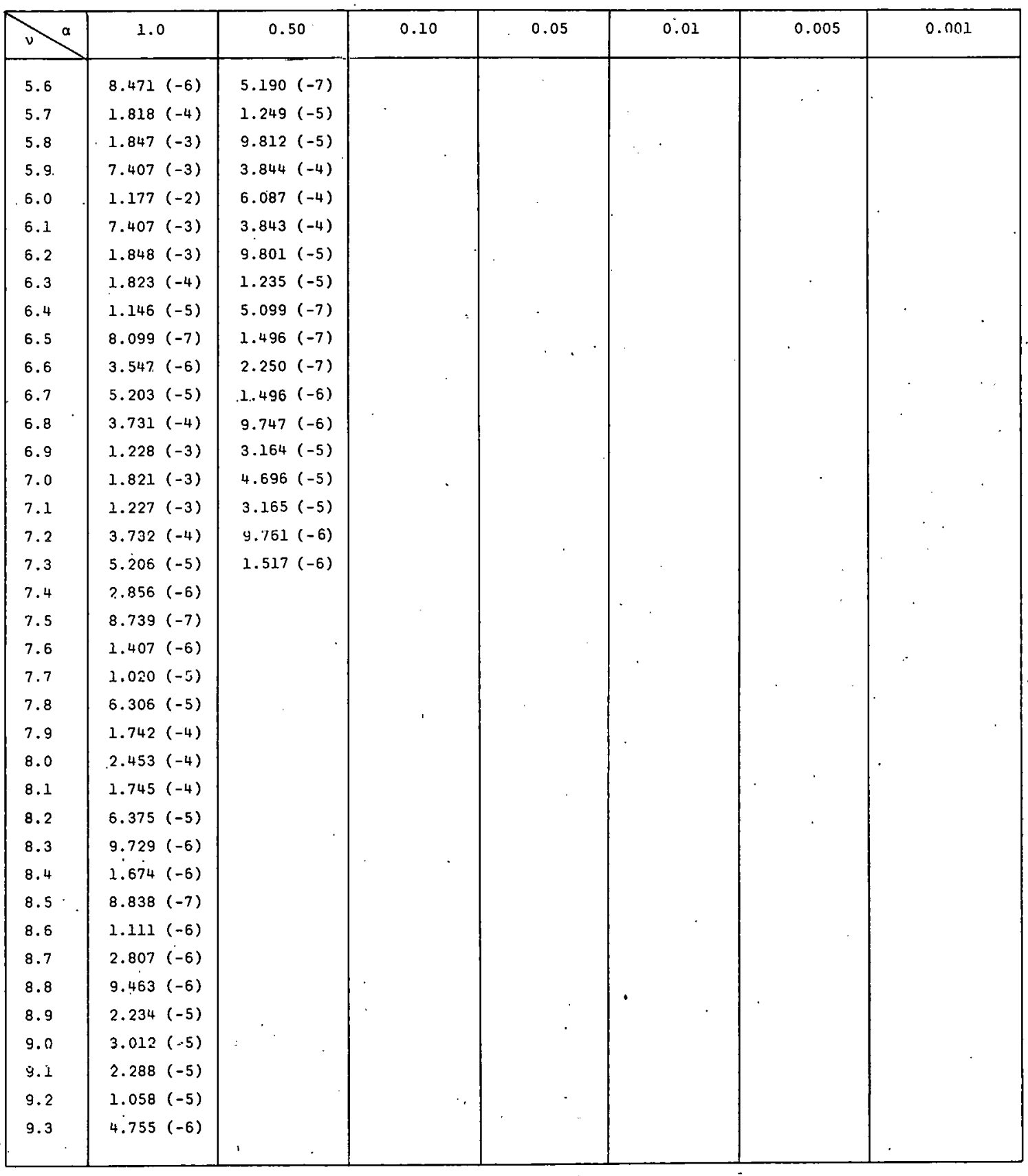


Table VI

THE DIFFERENCE FUNCTION FOR CASE IC

$E(v) \simeq D(v) \quad(v>1.5)$

$\Delta=0.012 \quad(r=0.02)$

\begin{tabular}{|c|c|c|c|c|c|c|c|}
\hline$v$ & 1.0 & 0.5 & 0.1 & 0.05 & 0.01 & 0.005 & 0.001 \\
\hline 1.0 & -29.720 & -18.499 & -4.474 & -2.291 & -0.466 & -0.233 & -0.049 \\
\hline 1.97 & $5.373(-1)$ & $4,429(-1)$ & $1.321(-1)$ & $5.946(-2)$ & $1.446(-2)$ & $7.266(-3)$ & $1.459(-3)$ \\
\hline 1.98 & 3.049 & 2.513 & $7.499(-1)$ & $3.942 \cdot(-1)$ & $8.205(-2)$ & $4.123(-2)$ & $8.279(-3)$ \\
\hline 1.99 & 8.640 & 7.123 & 2.125 & 1.1 .17 & $2.325(-1)$ & $1.168(-1)$ & $2.346(-2)$ \\
\hline 2.0 & 12.227 & 10.080 & 0.007 & 1. . 581 & $3.291(-1)$ & $1.654(-1)$ & $3.320(-2)$ \\
\hline 2.01 & 8.640 & 7.123 & 2.125 & 1.117 & $2.325(-1)$ & $1.168(-1)$ & $2.340(-2)$ \\
\hline 2.02 & 3.049 & 2.513 & $7.499(-1)$ & $3.942(-1)$ & $8.205(-2)$ & $4.123(-2)$ & $8.279(-3)$ \\
\hline 2.03 & $5.373(-1)$ & $4.429(-1)$ & $1.321(-1)$ & $6.946(-2)$ & $1.446(-2)$ & $7.266(-3)$ & $1.459(-3)$ \\
\hline 2.97 & $6.215(-1)$ & $2.562(-1)$ & $1.529(-2)$ & $4.018(-3)$ & $1.675(-4)$ & $4.213(-5)$ & $1.710(-6)$ \\
\hline 2.98 & 1.977 & $8.150(-1)$ & $4.864(-2)$ & $1.278(-2)$ & $5.324(-4)$ & $1.338(-4)$ & $5.391(-6)$ \\
\hline 2.99 & 3.960 & 1.632 & $9.740(-2)$ & $2.560(-2)$ & $1.066(-3)$ & $2.679(-4)$ & $1.077(-5)$ \\
\hline 3.00 & 4.991 & 2.057 & $1.228(-1)$ & $3.227(-2)$ & $1.343(-3)$ & $3.376(-4)$ & $1.357(-5)$ \\
\hline 3.01 & 3.960 & 1.632 & $9.740(-2)$ & $2.560(-2)$ & $1.066(-3)$ & $2.679(-4)$ & $1.077(-5)$ \\
\hline 3.02 & 1.977 & $8.150(-1)$ & $4.864(-2)$ & $1.278 \cdot(-2)$ & $5.323(-4)$ & $1.338(-4)$ & $5.391(-6)$ \\
\hline 3.03 & $6.215(-1)$ & $2.562(-1)$ & $1.529(-2)$ & $4.018(-3)$ & $1.674(-4)$ & $4.212(-5)$ & $1.708(-6)$ \\
\hline 3.97 & $3.020(-1)$ & $6.224(-2)$ & $7.437(-4)$ & $9.804(-5)$ & $8.978(-7)$ & & \\
\hline 3.98 & $7.194(-1)$ & $1.483(-1)$ & $1.770(-3)$ & $2.330(-4)$ & $2.020(-6)$ & & \\
\hline 3.99 & 1.211 & $2.496(-1)$ & $2.980(-3)$ & $3.919(-4)$ & $3.343(-6)$ & & \\
\hline 4.00 & 1.441 & $2.970(-1)$ & $3.544(-3)$ & $4.661(-4)$ & $3.960(-6)$ & & \\
\hline 4.01 & 1.211 & $2.496 \cdot(-1)$ & $2.980(-3)$ & $3.919(-4)$. & $3.344(-6)$ & & \\
\hline 4.02 & $7: 194 \cdot(-1)$ & $1.483(-1)$ & $1.770(-3)$ & $2.329 .(-4)$ & $2.020(-6)$ & & \\
\hline 4.03 & $3.020(-1)$ & $6.224(-2)$ & $.7 .436(-4)$ & $9.803 .(-5)$ & $8.058(-7)$ & & \\
\hline 4.97 & $9.229(-2)$ & $9.512(-3)$ & $2.326(-5)$ & $1.761(-6)$ & & & \\
\hline 4.98 & $1.848(-1)$ & $1.905(-2)$ & $4.601(-5)$ & $3.254(-6)$ & & & \\
\hline 4.99 & $2.803(-1)$ & $2.884(-2)$ & $6.950(-5)$ & $4.796(-6)$ & & & . \\
\hline 5.00 & $3.221(-1)$ & $3.331(-2)$ & $7.977(-5)$ & $5.469(-6)$ & . & & \\
\hline 5.01 & $2.803(-1)$ & $2.889(-2)$ & $6.951(-5)$ & $4.802(-6)$ & & & \\
\hline 5.02 & $1.848(-1)$ & $1.905(-2)$ & $4.501(-5)$ & $3.257(-6)$ & & & \\
\hline 5.03 & $9.228(-2)$ & $9.512(-3)$ & $2.326(-5)$ & $1.760(-6)$ & & & \\
\hline 5.94 & $9.140(-4)$ & $4.773(-5)$ & & & & & \\
\hline 5.96 & $9.235(-3)$ & $4.764(-4)$ & & & & & \\
\hline 5.98 & $3.703(-2)$ & $1.908(-3)$ & · & & & & \\
\hline 6.00 & $5.883(-2)$ & $3.032(-3)$ & & & & & \\
\hline 6.02 & $3.703(-2)$ & $1.908(-3)$ & & & & & \\
\hline 6.04 & $9.235(-3)$ & $4.764(-4)$ & & & & & \\
\hline 6.06 & $9.140(-4)$ & $4.773(-5)$ & & & & & \\
\hline 6.94 & $2.571(-4)$ & $7.334(-6)$ & & & & & \\
\hline 6.96 & $1.858(-3)$ & $4.858(-5)$ & $\cdot$ & & & & \\
\hline 6.98 & $6.105(-3)$ & $1.580(-4)$ & & & & & \\
\hline
\end{tabular}


Table VI (Contd.)

THE DIFFERENCE FUNCTION FOR CASE IC

$$
\begin{array}{rlrl}
E(v) & \simeq D(v) & & (v>1.5) \\
\Delta & =0.012 \quad \therefore \quad & (r=0.02)
\end{array}
$$

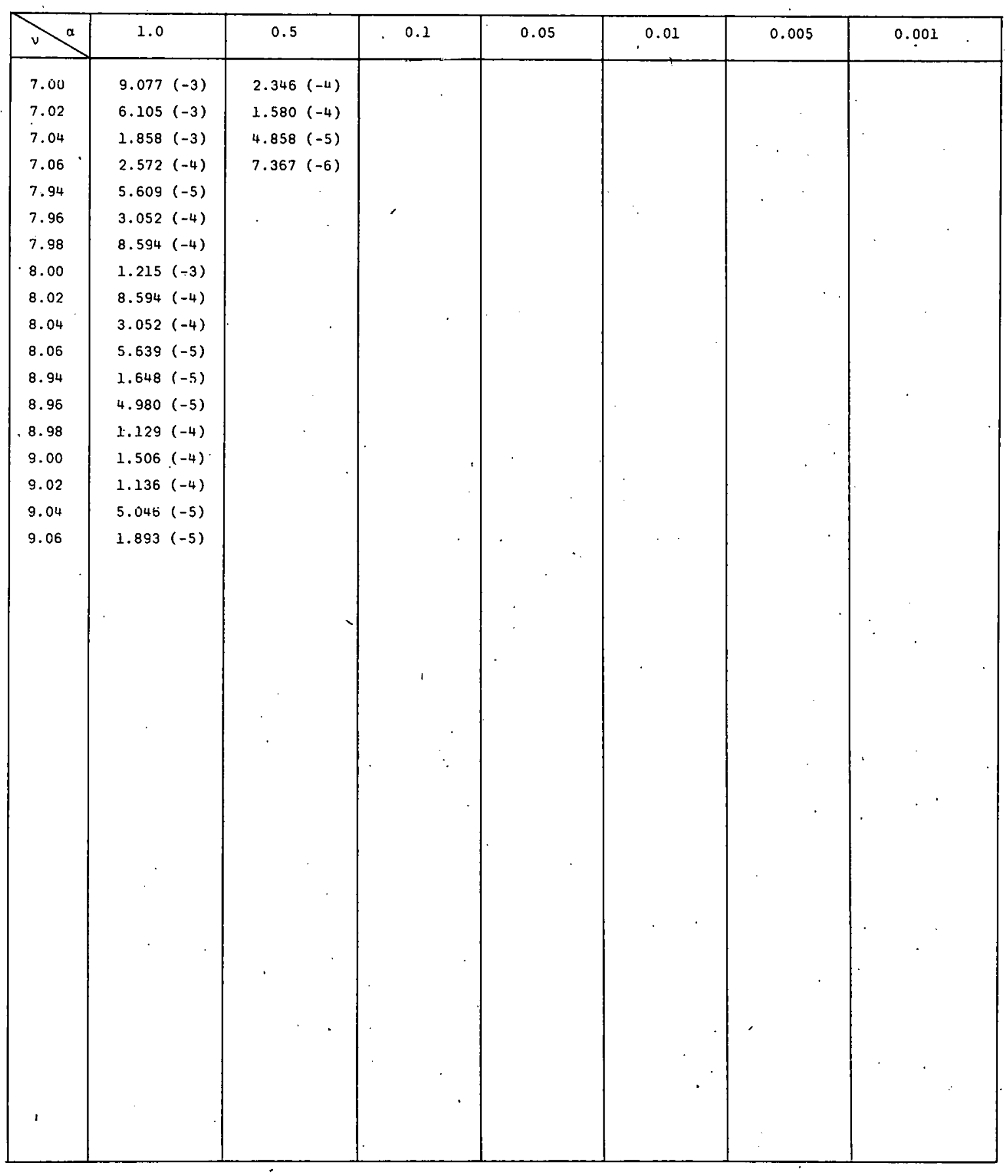




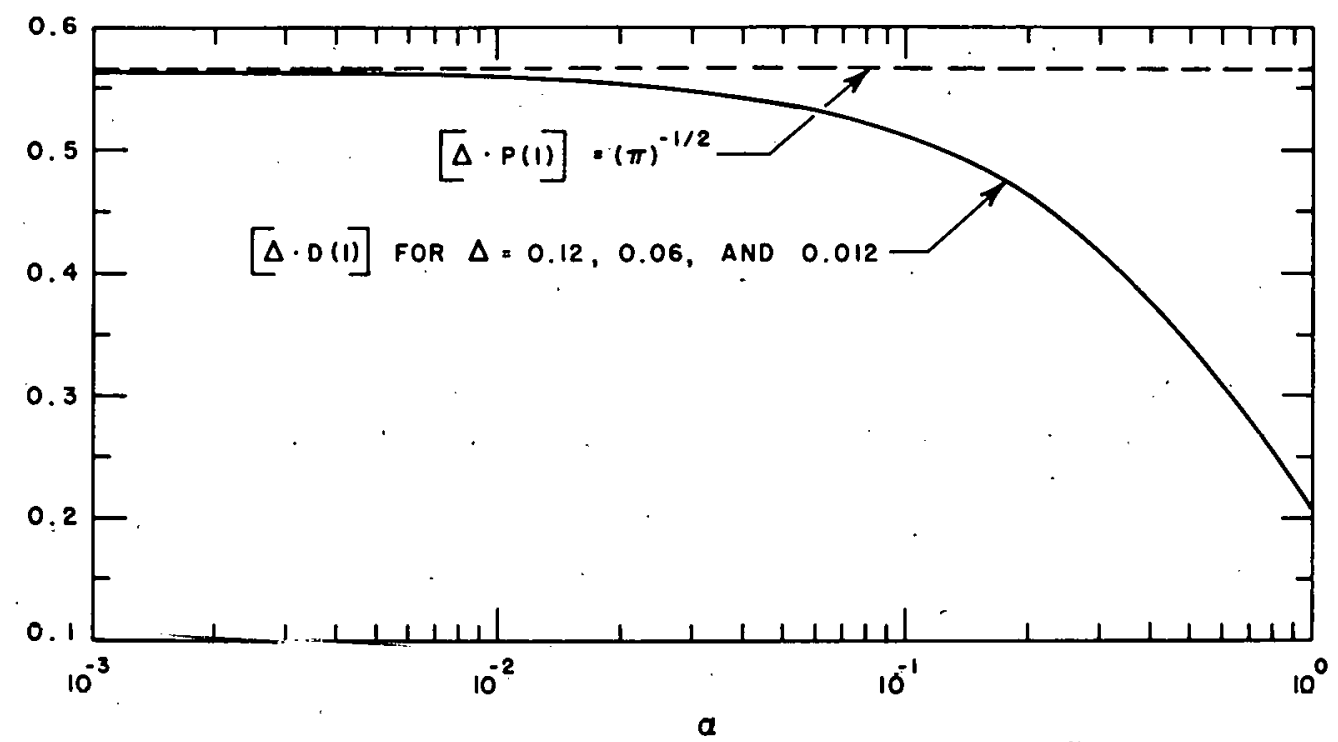

$112-4746$

Fig. 17. Comparison of $[\Delta \cdot D(1)]$ as a Function of $\alpha$ with the Limiting Value $[\Delta \cdot P(1)]=(\pi)^{-1 / 2}$ for $\Delta=0.12$, 0.06 , and 0.012

(2) The resolution of a given sum peak, $r_{n}$, is a linear function of $r$ or $\Delta$.

(3) For a given value of $\alpha$, the peak height of a given sum peak, $\mathrm{p}_{n}$, is a linear function of $\mathrm{r}^{-1}$ or $\Delta^{-1}$.

Application of these rules will facilitate interpolation of the present data. For example, Fig. 18 displays $r_{n}$, the resolution of the $n^{\text {th }}$ sum peak, as a function of $r$, for $n=2,3,4,5,6$, and 7 . In view of rule (1), these curves permit an estimate of the resolution for sum peaks up to $n=7$, for all $r \leq 0.20$. In addition, employing rule (3), together with the tabulated



$112-4748$
Fig. 18

Sum Peak Resolution $r_{n}$ as a Function of $r$, the Resolution of the Ideal Pulse-height Distribution, for the Sum Peaks $\mathrm{n}=2,3,4,5,6$, and 7 
data, permits interpolation of the peak height of a sum peak, $p_{n}$, for any $\alpha \leq 1$. Hence, determination of the two parameters $r_{n}$ and $p_{n}$, in this manner, will completely define the sum peak of interest.

\section{CONCLUSION}

The tabulations presented in this report can be used to obtain pileup estimates over the entire range of $\nu$ and $\alpha$ considered for each corresponding special case. In regions where $D(\nu)$ is not rapidly varying, graphical interpolation can be used to determine $D(\nu)$ to within a few percent. For rapidly varying $\mathrm{D}(\nu)$, as at integral values of $\nu$ in Case IIa, or in the neighborhood of sum peaks. in Case Ic, graphical interpolations of roughly 5-10\% accuracy should still be possible.

\section{ACKNOẄLEDGMENTS}

The author is indebted to Dr. E. F. Bennett and Dr. K. G. A. Porges for helpful discussions. In addition, the author gratefully acknowledges the assistance of Miss Ingeborg. Olson who wrote the computer programs used in the se calculations. 


\section{REFERENCES}

1. S. Rozen, Pile-Up Rejection Circuits, Nucl. Instr. Methods 11 , $316-320(1961)$.

2. M. G. Strauss, Solid State Pulse-Height Encoding System with Pile-Up Reduction for Counting at High Input Rates, Rev. Sci. Instr. 34, 335-345 (1963).

3. R. Segel, Reduction of Pile-Up Contamination in Pulse Height Spectra, TID-18300 (January 1963).

4. G. Mathé, Method for the Elimination of Superimposed Pulses in Nuclear Spectroscopy, Nucl. Instr. Methods 23, 261-263 (1963).

5. B. B. Rossi and H. H. Staub, Iunizatinn Chambers and Counters, McGraw-Hill, Inc., New York (1949), Appendix 11.

6. L. Pal, Application of the Theory of Stochastic Processes to the Investigation of Nuclear Fission, JETP 3 , 264-268 (1956).

7. B. Souček, Distribution Function for Output of Certain Linear Filters for Random Input Events, J. Electron. Contr. 16, 81-90 (1964).

8. B. Soucek, The Effect of a Random Noise Background Upon the Amplitude Distribution Function of Random Events, J. Electron. Contr. 16, 91-100 (1964).

9. B. Souček, Distribution Measurements Errors Due to the Pile-Up Effect, Nucl. Instr. Methods 28, 306-308 (1964).

10. W. Feller, An Introduction to Probability Theory and Its Applications, John Wiley \& Sons, Inc., New York (1.950), Vol. I, Chapt. XI.

11. O. R. Frisch, Fluctuations in Chain Processes, CRL-57, AECL No. 748 (1959).

12. H. Cramér, Mathematical Methods of Statistics, Princeton University Press, Princeton, N. J. (1946), Chapt. XV.

13. S. O. Rice, "Mathematical Analysis of Random Noise," Paper No. 4, p. 133, Selected Papers on Noise and Stochastic Processes, Edited by N. Wax, Dover Publications, Inc., New York (1954). 